\title{
Heavy flavour production at Tevatron and parton shower effects
}

\author{
H. Jung, ${ }^{a, b}$ M. Kraemer, ${ }^{a}$ A.V. Lipatov, ${ }^{c}$ and N.P. Zotov ${ }^{c}$ \\ ${ }^{a}$ DESY, \\ Notkestr 85, Hamburg, FR, Germany \\ ${ }^{b}$ University Antwerp, \\ Groenenborgerlaan 17, Antwerp, Belgium \\ ${ }^{c}$ D. V. Skobeltsyn Institute of Nuclear Physics, M. V. Lomonosov Moscow State University, \\ Leninskie Gory, 1, 119991 Moscow, Russia \\ E-mail: hannes.jung@desy.de, mira.kraemer@desy.de, \\ lipatov@theory.sinp.msu.ru, zotov@theory.sinp.msu.ru
}

Abstract: We present hadron-level predictions from the Monte Carlo generator CASCADE and numerical calculations of charm and beauty production at the Fermilab Tevatron within the framework of the $k_{T}$-factorization QCD approach. Our consideration is based on the CCFM-evolved unintegrated gluon densities in a proton. The performed analysis covers the total and differential cross sections of open charm and beauty quarks, $B$ and $D$ mesons (or rather muons from their semileptonic decays) and the total and differential cross sections of $b \bar{b}$ di-jet hadroproduction. We study the theoretical uncertainties of our calculations and investigate the effects coming from parton showers in initial and final states. Our predictions are compared with the recent experimental data taken by the D0 and CDF collaborations. Special attention is put on the specific angular correlations between the final-state particles. We demonstrate that the final state parton shower plays a crucial role in the description of such observables. The decorrelated part of angular separations can be fully described, if the process $g g^{*} \rightarrow g g$ is included.

Keywords: Phenomenological Models, Heavy Quark Physics, Hadronic Colliders, QCD

ARXIV EPRINT: 1009.5067 


\section{Contents}

1 Introduction $\quad 1$

2 Theoretical framework 3

3 Numerical results $\quad 5$

3.1 Inclusive charm and beauty production 5

$\begin{array}{lll}3.2 & b \bar{b} \text { di-jet production } & 11\end{array}$

$\begin{array}{lll}3.3 & \text { Parton shower effects and additional gluon processes } & 13\end{array}$

4 Conclusions $\quad 16$

\section{Introduction}

Charm and beauty production at high energies is subject of intense studies from both theoretical and experimental points of view [1-6]. From the theoretical point, these processes provide an opportunity to test the different predictions based on Quantum Chromodynamics (QCD) since the dominant production mechanism at high energies (i.e. small $x$ ) is believed to be quark pair production through the gluon-gluon fusion subprocess. At present, the problem of description of the charm and beauty production at the Tevatron within QCD is not fully solved. So, the difference between the D0 and CDF measurements [1-4] of the $b$-quark and $B$-meson production cross sections and the calculations $[7,8]$ performed in the framework of fixed-order next-to-leading logarithm scheme (FONLL) is about of a factor of 1.7. The central FONLL predictions [9] also lie below the data on the $D^{0}$, $D^{+}, D^{*+}$ and $D_{s}^{+}$cross sections which has been measured [5] by the CDF collaboration as a functions of their transverse momenta. Recently the calculations in the general-mass variable-flavour-number scheme (GM-VFNS) were performed [10, 11] for the transverse momentum distribution of $B$ and $D$ mesons and have been found to be consistent with the data $[4,5]$. However, there is still no GM-VFNS predictions for other measured quantities (like distributions in rapidity and azimuthal angle difference between the momenta of final mesons).

Heavy flavour production has been considered [12-18] also in the framework of the $k_{T}$-factorization QCD approach [19-22]. This approach is based on the Balitsky-FadinKuraev-Lipatov (BFKL) [23-25] or Ciafaloni-Catani-Fiorani-Marchesini (CCFM) [26-29] equations for the non-collinear gluon evolution in a proton and gives the possibility to take into account large logarithmic terms (proportional to $\ln 1 / x$ and, in the case of CCFM, also to $\ln 1 /(1-x)$ ). A detailed description and discussion of the $k_{T}$-factorization approach can be found in [30-32]. A reasonable agreement between the $k_{T}$-factorization predictions and the Tevatron data on the heavy flavour production has been found in [13-18]. It 
was demonstrated $[12,17,18]$ that studying the specific angular correlations between the transverse momenta of produced quarks can give an unique information about the noncollinear gluon evolution in a proton since taking into account the non-vanishing initial gluon transverse momentum $\mathbf{k}_{T}$ in the $k_{T}$-factorization approach leads to the violation of back-to-back kinematics event at leading order.

Measurements of $b \bar{b}$ di-jet total and differential cross sections (as a functions of the leading jet transverse energy $E_{T}$ and the di-jet invariant mass) and azimuthal angle correlations between two $b$-jets have been performed by the CDF collaboration [33, 34]. These measurements significantly extend the energy range investigated by previous analyses $[1-5]$. Studying the $b$-jet production is specially interesting since there are no additional assumptions on the fragmentation of the beauty quark into the $B$ meson. The CDF collaboration has reported the preliminary data [6] on the charm pair production, where the $D^{0}, D^{*-}$ pair cross section and the $D^{+}, D^{*-}$ pair cross section as a function of the azimuthal angle between two charmed mesons have been meausred. The angular correlations in $B$-meson production have been also measured [1-3] by the D0 and CDF collaborations.

The main goal of present paper is to give a systematic analysis of all available experimental data $[1-6,33,34]$ on the heavy flavour production at the Tevatron in the framework of the $k_{T}$-factorization formalism. We produce the relevant numerical calculations in two ways. First, we will perform analytical parton-level calculations (labeled as LZ in the following) similar to that done in $[17,18]$. In these calculations we will use the CCFM-evolved gluon densities [35] as default sets. The measured cross sections of heavy quark production will be compared with the predictions of Monte Carlo event generator CASCADE [36, 37]. CASCADE is a full hadron level Monte Carlo event generator for $e p, \gamma p, p \bar{p}$ and $p p$ processes, which uses the CCFM evolution equation for the initial state cascade in a backward evolution approach supplemented with off-shell matrix elements for the hard scattering. ${ }^{1}$ In this way we will investigate the influence of parton showers in initial and final states for the description of the data. We will study the possible sources of theoretical uncertainties of our predictions (i.e. uncertainties connected with the gluon evolution scheme, heavy quark mass, hard scale of partonic subprocess and the heavy quark fragmentation functions). To investigate the dependence of our predictions on the non-collinear evolution scheme we will apply the unintegrated gluon densities derived from the usual (DGLAP-evolved) parton distributions (in the Kimber-Martin-Ryskin (KMR) [39, 40] approximation). Our special goal is to study specific kinematic properties of the final heavy quark-antiquark pair which are strongly related to the non-zero initial gluon transverse momentum.

The outline of our paper is following. In section 2 we recall shortly the basic formulas of the $k_{T}$-factorization approach with a brief review of calculation steps. In section 3 we present the numerical results of our calculations and a discussion. Section 4 contains our conclusions.

\footnotetext{
${ }^{1} \mathrm{~A}$ discussion of the phenomenological applications of CASCADE can be found in [38].
} 


\section{Theoretical framework}

The main formulas have been obtained previously in [16-18]. Here we only recall some of them. The cross section of heavy quark hadroproduction at high energies in the $k_{T^{-}}$ factorization approach is calculated as a convolution of the off-shell (i.e. $k_{T}$-dependent) partonic cross section $\hat{\sigma}$ and the unintegrated gluon distributions in a proton. It can be presented in the following form:

$$
\begin{aligned}
\sigma(p \bar{p} \rightarrow Q \bar{Q} X)=\int \frac{1}{16 \pi\left(x_{1} x_{2} s\right)^{2}} \mathcal{A}\left(x_{1}, \mathbf{k}_{1 T}^{2}, \mu^{2}\right) & \mathcal{A}\left(x_{2}, \mathbf{k}_{2 T}^{2}, \mu^{2}\right)\left|\overline{\mathcal{M}}\left(g^{*} g^{*} \rightarrow Q \bar{Q}\right)\right|^{2} \times \\
& \times d \mathbf{p}_{1 T}^{2} d \mathbf{k}_{1 T}^{2} d \mathbf{k}_{2 T}^{2} d y_{1} d y_{2} \frac{d \phi_{1}}{2 \pi} \frac{d \phi_{2}}{2 \pi}
\end{aligned}
$$

where $\mathcal{A}\left(x, \mathbf{k}_{T}^{2}, \mu^{2}\right)$ is the unintegrated gluon distribution in a proton, $\left|\overline{\mathcal{M}}\left(g^{*} g^{*} \rightarrow Q \bar{Q}\right)\right|^{2}$ is the off-shell (i.e. depending on the initial gluon virtualities $\mathbf{k}_{1 T}^{2}$ and $\mathbf{k}_{2 T}^{2}$ ) matrix element squared and averaged over initial gluon polarizations and colors, and $s$ is the total center-ofmass energy. The produced heavy quark $Q$ and anti-quark $\bar{Q}$ have the transverse momenta $\mathbf{p}_{1 T}$ and $\mathbf{p}_{2 T}$ and the center-of-mass rapidities $y_{1}$ and $y_{2}$. The initial off-shell gluons have a fraction $x_{1}$ and $x_{2}$ of the parent protons longitudinal momenta, non-zero transverse momenta $\mathbf{k}_{1 T}$ and $\mathbf{k}_{2 T}\left(\mathbf{k}_{1 T}^{2}=-k_{1 T}^{2} \neq 0, \mathbf{k}_{2 T}^{2}=-k_{2 T}^{2} \neq 0\right)$ and azimuthal angles $\phi_{1}$ and $\phi_{2}$. The analytic expression for the $\left|\overline{\mathcal{M}}\left(g^{*} g^{*} \rightarrow Q \bar{Q}\right)\right|^{2}$ can be found, for example, in $[16,19-22]$.

The unintegrated gluon distribution in a proton $\mathcal{A}\left(x, \mathbf{k}_{T}^{2}, \mu^{2}\right)$ in (2.1) can be obtained from the analytical or numerical solution of the BFKL or CCFM evolution equations. In the numerical calculations we have tested a few different sets. First of them (A0) was obtained in [35] from the CCFM evolution equation. The initial (starting) distribution $\mathcal{A}_{0}\left(x, \mathbf{k}_{T}^{2}, \mu_{0}^{2}\right)$ has been used in the following form:

$$
x \mathcal{A}_{0}\left(x, \mathbf{k}_{T}^{2}, \mu_{0}^{2}\right)=N x^{p_{0}}(1-x)^{p_{1}} \exp \left(-\mathbf{k}_{T}^{2} / k_{0}^{2}\right),
$$

where all input parameters have been fitted to describe the proton structure function $F_{2}\left(x, Q^{2}\right)$. An equally good fit can be obtained using different values for the soft cut and a different value for the width of the intrinsic $\mathbf{k}_{T}$ distribution (the CCFM set B0). A reasonable description of the $F_{2}$ data can be achieved [35] by both these sets.

To evaluate the unintegrated gluon densities in a proton $\mathcal{A}\left(x, \mathbf{k}_{T}^{2}, \mu^{2}\right)$ we apply also the KMR approach [39, 40]. The KMR approach is a formalism to construct the unintegrated parton (quark and gluon) distributions from the known conventional parton distributions $x a\left(x, \mu^{2}\right)$, where $a=g$ or $a=q$. In this scheme, the unintegrated gluon distribution is given by the expression

$$
\begin{aligned}
\mathcal{A}\left(x, \mathbf{k}_{T}^{2}, \mu^{2}\right)= & T_{g}\left(\mathbf{k}_{T}^{2}, \mu^{2}\right) \frac{\alpha_{s}\left(\mathbf{k}_{T}^{2}\right)}{2 \pi} \times \\
& \times \int_{x}^{1} d z\left[\sum_{q} P_{g q}(z) \frac{x}{z} q\left(\frac{x}{z}, \mathbf{k}_{T}^{2}\right)+P_{g g}(z) \frac{x}{z} g\left(\frac{x}{z}, \mathbf{k}_{T}^{2}\right) \Theta(\Delta-z)\right],
\end{aligned}
$$




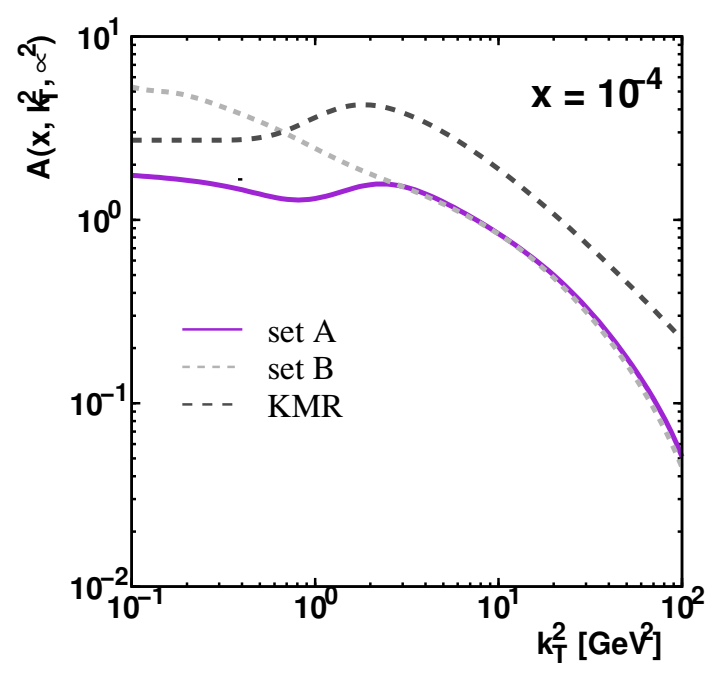

(a)

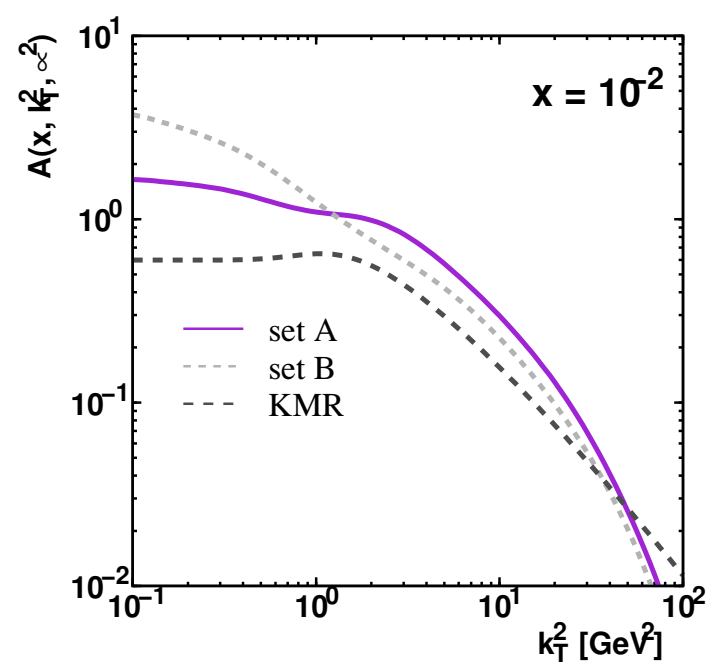

(c)

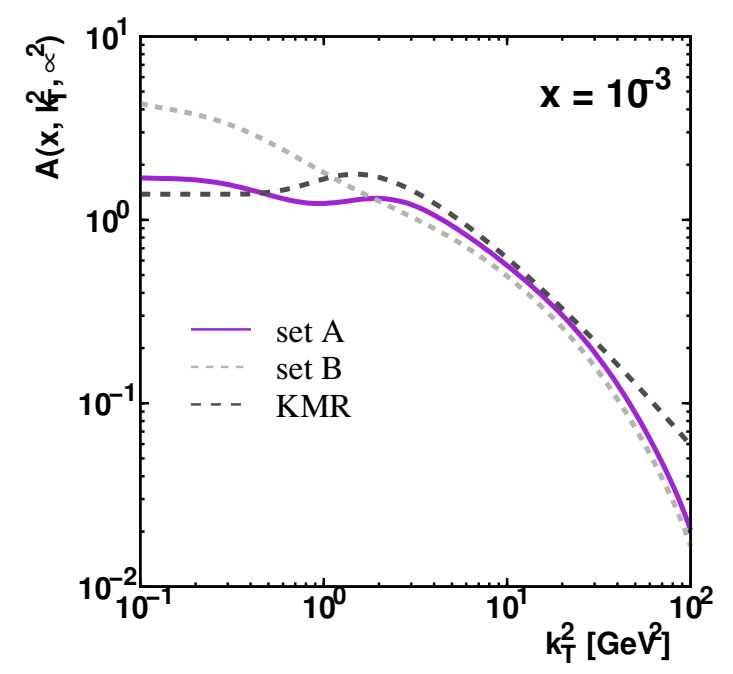

(b)

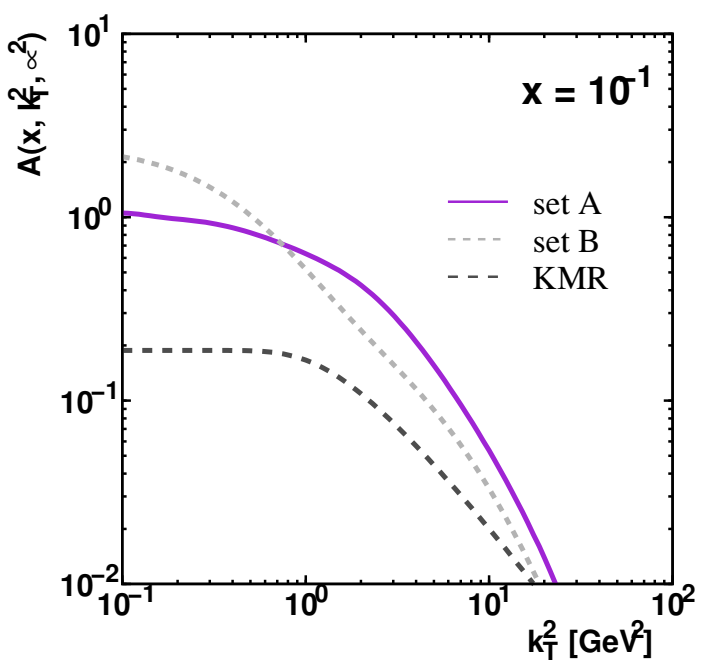

(d)

Figure 1. The unintegrated gluon densities in a proton $\mathcal{A}\left(x, \mathbf{k}_{T}^{2}, \mu^{2}\right)$ as a function of $\mathbf{k}_{T}^{2}$ at $\mu^{2}=100 \mathrm{GeV}^{2}$. The solid, dashed and dotted curves correspond to the CCFM set A0, CCFM set $\mathrm{B} 0$ and KMR distributions, respectively.

where $P_{a b}(z)$ are the usual unregulated leading order DGLAP splitting functions, $q\left(x, \mu^{2}\right)$ and $g\left(x, \mu^{2}\right)$ are the conventional quark and gluon densities ${ }^{2}$ and $T_{g}\left(\mathbf{k}_{T}^{2}, \mu^{2}\right)$ is the Sudakov form factor. The theta function $\Theta(\Delta-z)$ implies the angular-ordering constraint $\Delta=$ $\mu /\left(\mu+\left|\mathbf{k}_{T}\right|\right)$ specifically to the last evolution step to regulate the soft gluon singularities.

In figure 1 we plot the CCFM set A0 (as a solid lines), the CCFM set B0 (as a dashed lines) and KMR (as a dotted lines) unintegrated gluon densities at probing scale $\mu^{2}=$ $100 \mathrm{GeV}^{2}$ as a function of $\mathbf{k}_{T}^{2}$ for different values of $x$. One can see that the shapes of gluon densities under consideration are very different from each other. In the following we will study the possible manifestations in the total and differential heavy flavour cross sections.

\footnotetext{
${ }^{2}$ Numerically, we have used the standard GRV 94 (LO) [41, 42], MSTW 2008 (LO) [43] (in LZ calculations) and MRST 99 [44] (CASCADE) sets.
} 
In our analytical calculations (LZ) the multidimensional integrations in eq. (2.1) have been performed by the means of Monte Carlo technique, using the routine vEGAS [45]. The full $\mathrm{C}++$ code is available from the authors on request.

\section{$3 \quad$ Numerical results}

We now are in a position to present our numerical results. First we describe our input and the kinematic conditions. After we fixed the unintegrated gluon distributions, the cross section (2.1) depends on the renormalization and factorization scales $\mu_{R}$ and $\mu_{F}$. In the numerical calculations we set $\mu_{R}^{2}=m_{Q}^{2}+\left(\mathbf{p}_{1 T}^{2}+\mathbf{p}_{2 T}^{2}\right) / 2, \mu_{F}^{2}=\hat{s}+\mathbf{Q}_{T}^{2}$ (where $\mathbf{Q}_{T}$ is the transverse momentum of initial off-shell gluon pair), $m_{c}=1.4 \pm 0.1 \mathrm{GeV}, m_{b}=$ $4.75 \pm 0.25 \mathrm{GeV}$ and use the LO formula for the coupling constant $\alpha_{s}\left(\mu^{2}\right)$ with $n_{f}=4$ active quark flavours at $\Lambda_{\mathrm{QCD}}=200 \mathrm{MeV}$, such that $\alpha_{s}\left(M_{Z}^{2}\right)=0.1232$.

\subsection{Inclusive charm and beauty production}

We begin the discussion by studying the role of non-zero gluon transverse momentum $k_{T}$ in the off-shell matrix elements involved in (2.1). In figure 2 we plot the differential cross section for $c \bar{c}$ and $b \bar{b}$ pair production as a function of transverse momentum $\mathbf{p}_{T}^{Q}$, rapidity $y^{Q}$ and the azimuthal angle difference between the transverse momenta of produced quarks $\Delta \phi^{Q Q}$ at $\sqrt{s}=1960 \mathrm{GeV}$. The solid histograms correspond to the results obtained according to the master formula in eq. (2.1). The dotted histograms are obtained by using the same formula but without virtualities of the incoming gluons in partonic amplitude and with the additional requirement $\mathbf{k}_{1,2}^{2}<\mu_{R}^{2}$. There are no cuts applied on the phase space of the produced quarks. As it was expected, in the back-to-back region $\Delta \phi^{Q Q} \sim \pi$ both results coincide with each other. However, we find that a sizeable effect appears at low $\Delta \phi^{Q Q}$. Therefore the non-zero gluon transverse momentum in the hard matrix element is important for the description of the data at low and mediate $\Delta \phi^{Q Q}$. This effect is more significant for charm production due to smaller $x$. We have checked that the predictions between the LZ and CASCADE calculations agree well at parton level.

Now we turn to the transverse momentum distributions of charm and beauty production. In the case of inclusive $b$-quark production, the transverse momentum distribution has been measured [1] by the D0 collaboration and has been presented in form of integrated cross section (as a function of $b$-quark minimal transverse momentum $p_{T \text { min }}^{b}$ ) at the total $p \bar{p}$ energy $\sqrt{s}=1800 \mathrm{GeV}$ for $\left|y^{b}\right|<1$ (note that there is no cut on the rapidity of $\bar{b}$ ). Our predictions are shown in figure 3 and compared to the data. We find good agreement between the LZ and CASCADE predictions. We find a good description of the data when using the CCFM-evolved (A0) gluon distribution. The results obtained by using the KMR gluon density are rather close to the NLO pQCD ones [46] (not shown) but lie below the data. The difference between the CCFM and KMR predictions comes from the different behaviour of these gluon densities (see figure 1) which is due to absence of small- $x$ resummation in the KMR distributions.

The CDF collaboration has measured $[4,5]$ the transverse momentum distributions of $B^{+}$and several $D$ mesons (namely, $D^{0}, D^{+}, D^{*+}$ and $D_{s}^{+}$) with $|y|<1$ (where $y$ is 


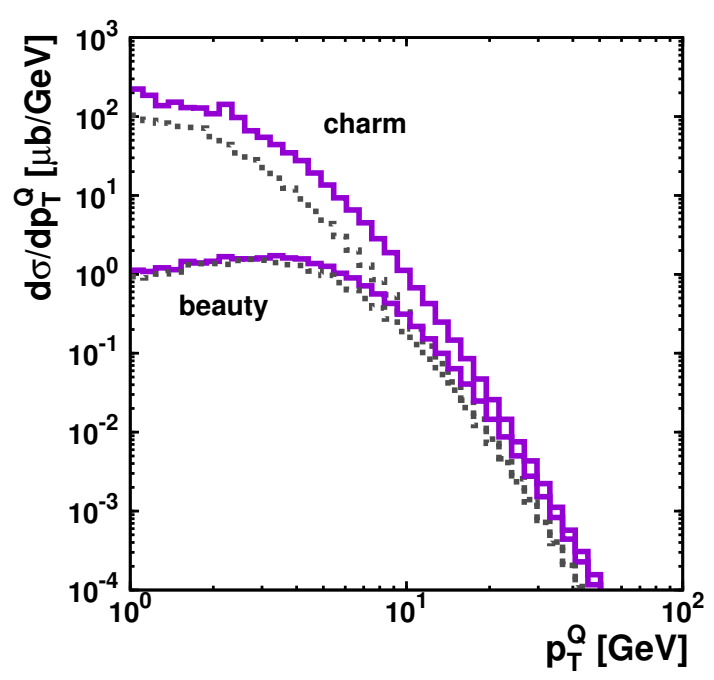

(a)

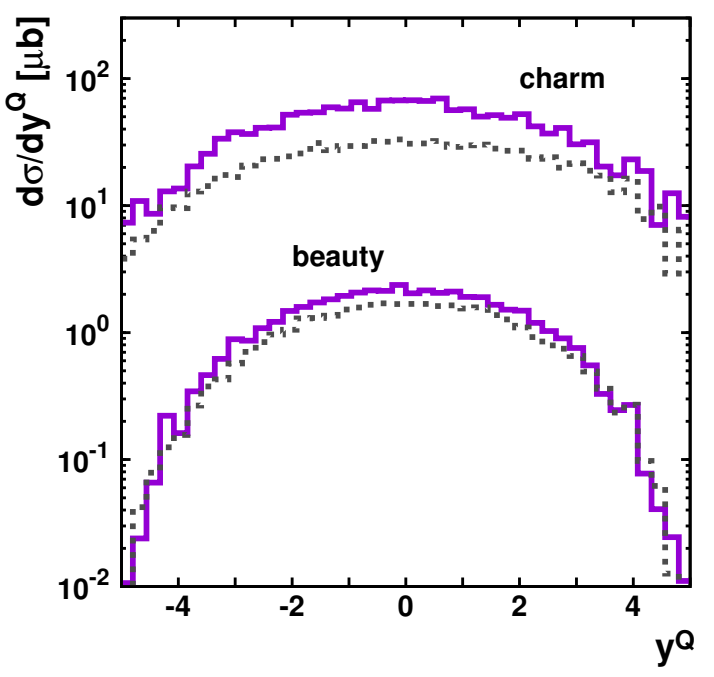

(b)

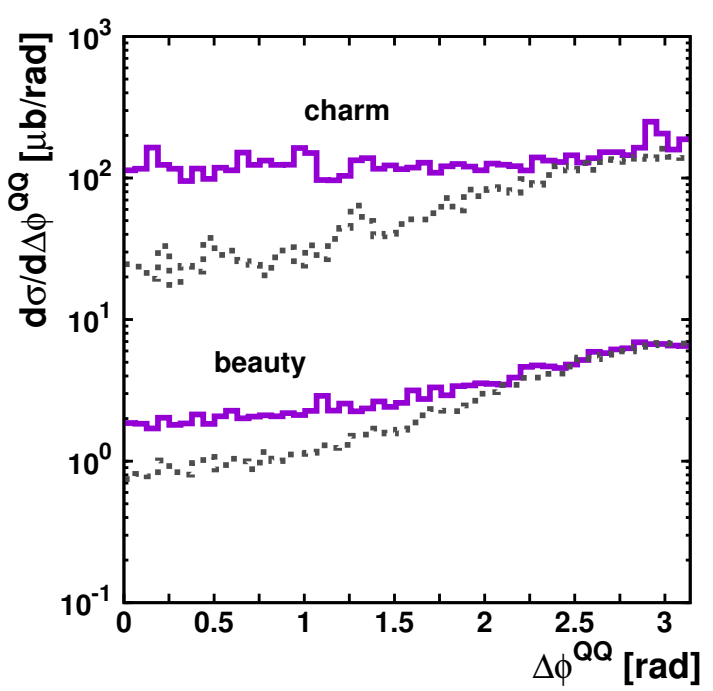

(c)

Figure 2. Importance of non-zero transverse momentum of incoming gluons in charm and beauty production at the Tevatron. The solid histograms correspond to the results obtained according to the master formula (2.1). The dotted histograms are obtained by using the same formula but now we switch off the virtualities of both incoming gluons in partonic amplitude and apply an additional requirement $\mathbf{k}_{1,2 T}^{2}<\mu_{R}^{2}$.

the meson rapidities in the center-of-mass frame) at $\sqrt{s}=1960 \mathrm{GeV}$. Our predictions are shown in figures 4-6 in comparison with the data $[4,5]$. The fragmentation of the charm and beauty quarks into a $B$ and $D$ mesons is described with the Peterson fragmentation function [47] with $\epsilon_{b}=0.006$ and $\epsilon_{c}=0.06$. According to [48, 49], the following branching fractions are used: $f\left(b \rightarrow B^{+}\right)=0.424, f\left(c \rightarrow D^{0}\right)=0.582, f\left(c \rightarrow D^{+}\right)=0.268$, $f\left(c \rightarrow D^{*+}\right)=0.229$ and $f\left(c \rightarrow D_{s}^{+}\right)=0.084$. The observed difference between the LZ and CASCADE predictions is due to the missing parton shower effects in the LZ calculations. We address this point in more detail in section 3.3 . 


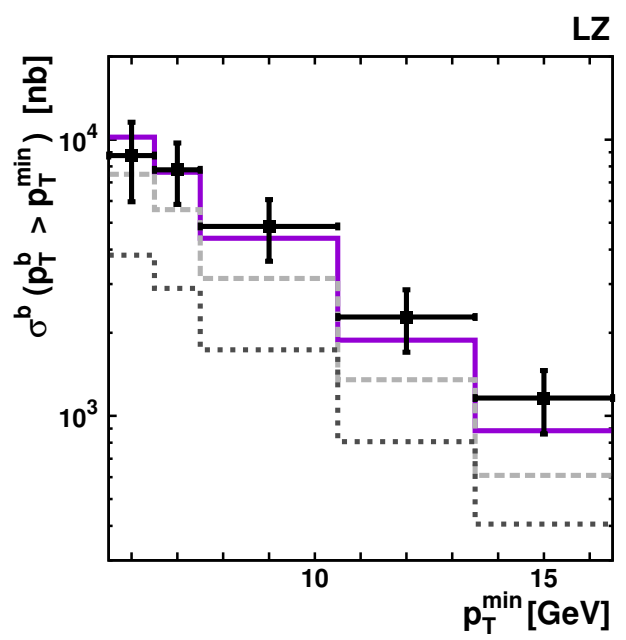

(a)

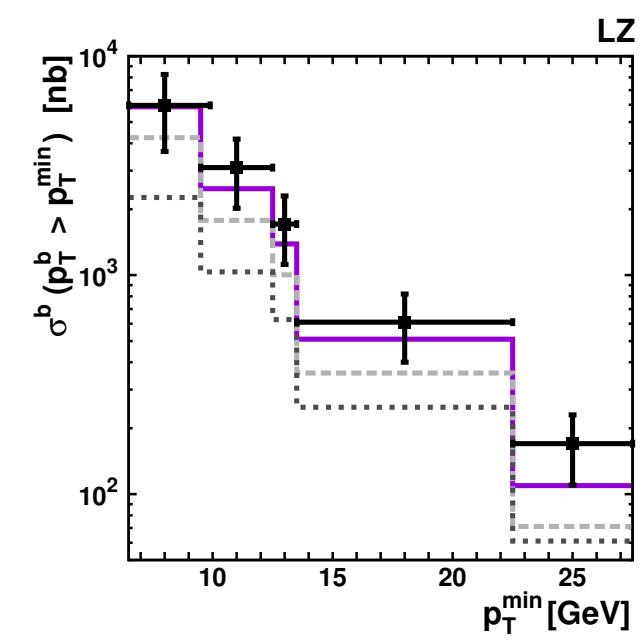

(c)

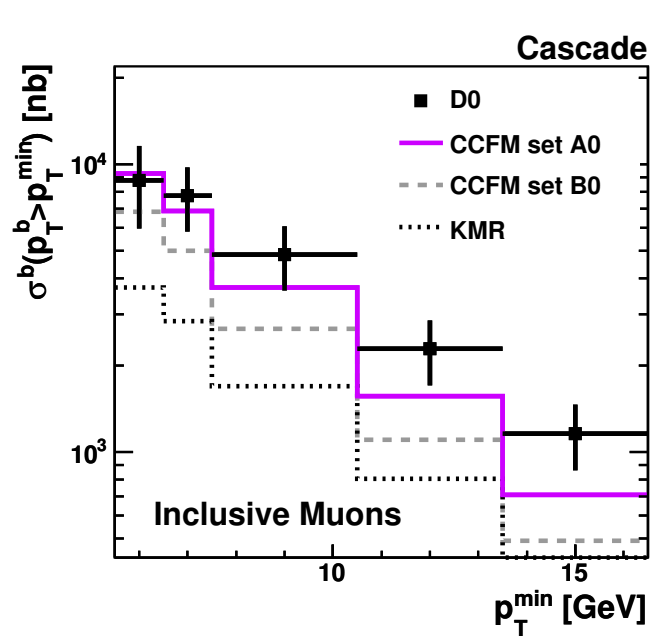

(b)

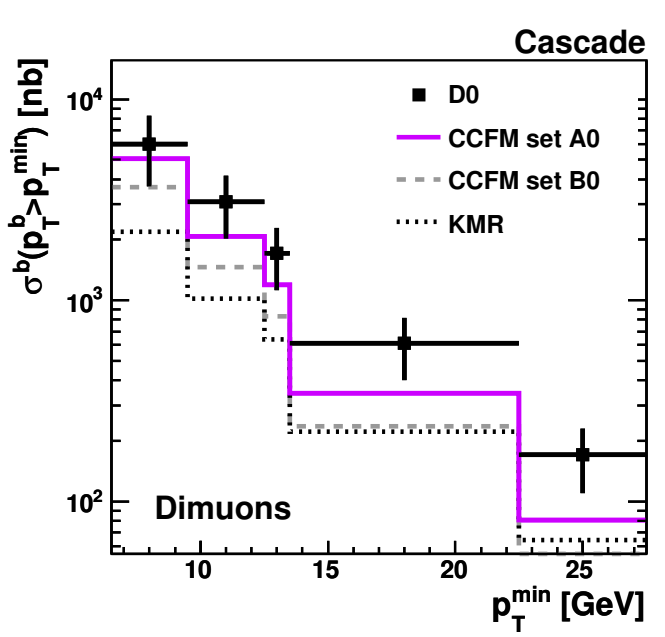

(d)

Figure 3. The transverse momentum distributions of open $b$-quark production at the Teavtron. The kinematical cuts applied are described in the text. The solid, dashed and dotted histograms correspond to the results obtained with the CCFM set A0, CCFM set B0 and KMR unintegrated gluon densities. The first column shows the LZ results while the second one depicts the CASCADE predictions. The experimental data are from D0 [1].

Since the predicted meson transverse momentum distributions depend on the quark-tohadron fragmentation, we have repeated our calculations for $B^{+}$and $D^{0}$ mesons with the shifted values of the Peterson shape parameter $\epsilon$, namely $\epsilon_{b}=0.003$ and $\epsilon_{c}=0.03$. These values are also often used in the NLO pQCD calculations. Additionally, we have applied the non-perturbative fragmentation functions which have been proposed in [7-9, 50] and which have been used in the FONLL calculations. The input parameters were determined $[9,50]$ by a fit to LEP data. The results of our calculations are shown in figure 7 . We find that the predicted cross sections (in the considered $p_{T}$ region) are larger for smaller values of parameter $\epsilon$ or if the fragmentation function from $[7-9,50]$ is used. However, the typical 


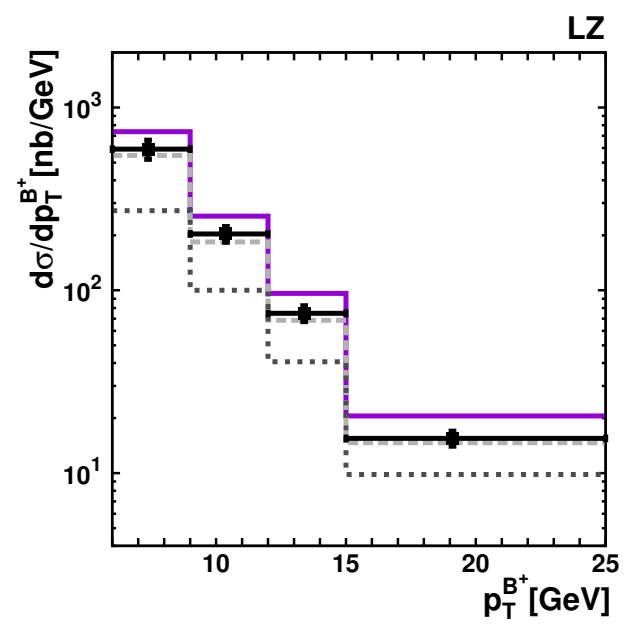

(a)

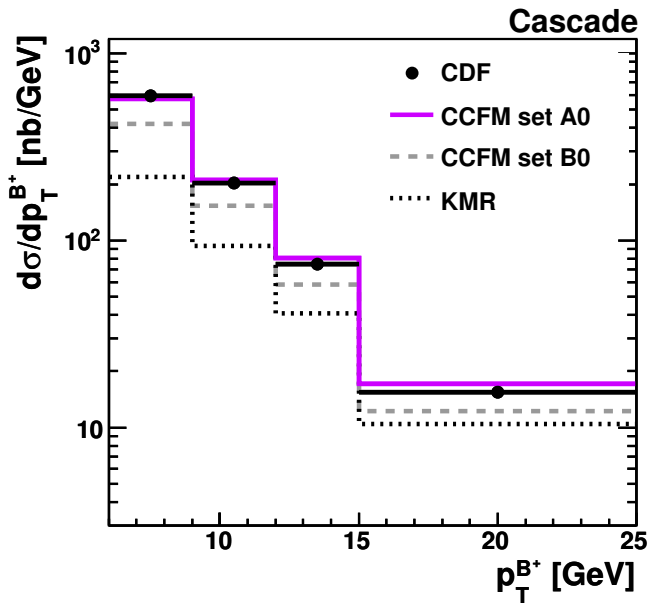

(b)

Figure 4. The cross section as a function of transverse momentum of $B^{+}$meson hadroproduction. The kinematical cuts applied are described in the text. The left histogram shows the LZ numerical results while the right plot depicts the CASCADE predictions. Notation of all histograms is the same as in figure 3. The experimental data are from CDF [4].

dependence of numerical predictions on the fragmentation scheme is much smaller than the dependence on the unintegrated gluon density.

The D0 experiment has measured muons originating from the semileptonic decays of $b$-quarks at $\sqrt{s}=1800 \mathrm{GeV}[1]$ for $4<p_{T}^{\mu}<25 \mathrm{GeV},\left|\eta^{\mu}\right|<0.8$ (for both muons) and $6<$ $m^{\mu \mu}<35 \mathrm{GeV}$, where $\eta^{\mu}$ is the muon pseudo-rapidity and $m^{\mu \mu}$ is the invariant mass of the produced muon pair. In [2] the measurements are extended to the forward muon rapidity region, namely $2.4<\left|y^{\mu}\right|<3.2$. To produce muons from $b$-quarks in the LZ calculations, we first convert $b$-quarks into $B$-mesons (using the Peterson fragmentation function with default value $\left.\epsilon_{b}=0.006\right)$ and then simulate their semileptonic decay according to the standard electroweak theory. The branching of $b \rightarrow \mu$ as well as the cascade decay $b \rightarrow c \rightarrow$ $\mu$ are taken into account with the branching fraction taken from [49]. The predictions of the LZ and CASCADE calculations are shown in figures 8 and 9. We find that our predictions with both CCFM-evolved unintegrated gluon densities describe the experimental data for both the transverse momentum and rapidity distributions of muons reasonably well.

The calculated total cross sections of the $b$-quarks, $B$ and $D$ mesons and their decay muons compared to the CDF experimental data [4,5] are listed in table 1 . In table 2 the systematic uncertainties of our calculations are summarized. To estimate the uncertainty coming from the renormalization scale $\mu_{R}$, we used the CCFM set $\mathrm{A} 0+$ and $\mathrm{A} 0$ - instead of the default density function A0 in CASCADE. These two sets represent a variation of the scale used in $\alpha_{s}$ in the off-shell matrix element. The A0+ stands for a variation of $2 \mu_{R}$, while set A0- reflects $\mu_{R} / 2$. In all heavy flavour analyses studied here, we observe a deviation of roughly $+10 \%$ for set $\mathrm{A} 0+$. The uncertainty coming from set A0- is generally smaller, but still positive. The dependence on the heavy flavour masses is investigated as well using CASCADE. We varied our default values of $m_{b}=4.75 \mathrm{GeV}$ by $\pm 0.25 \mathrm{GeV}$ and 


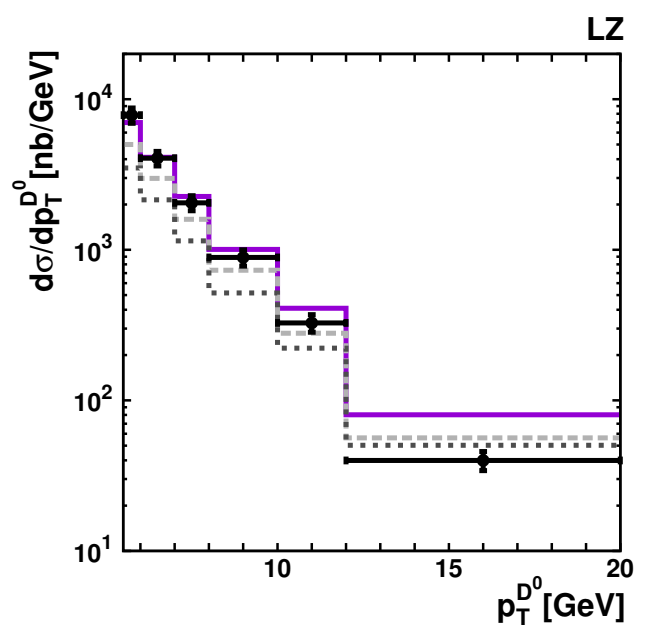

(a)

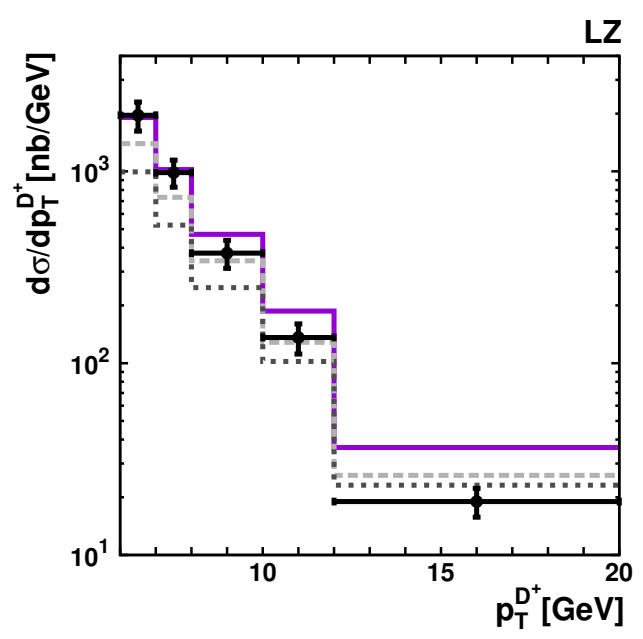

(c)

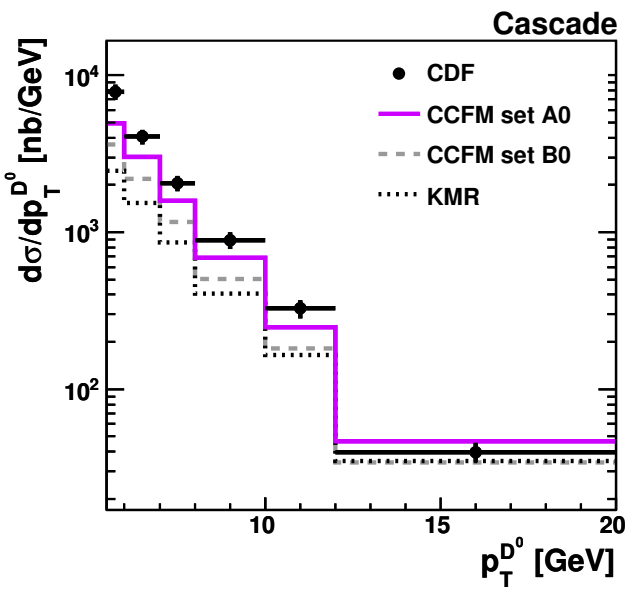

(b)

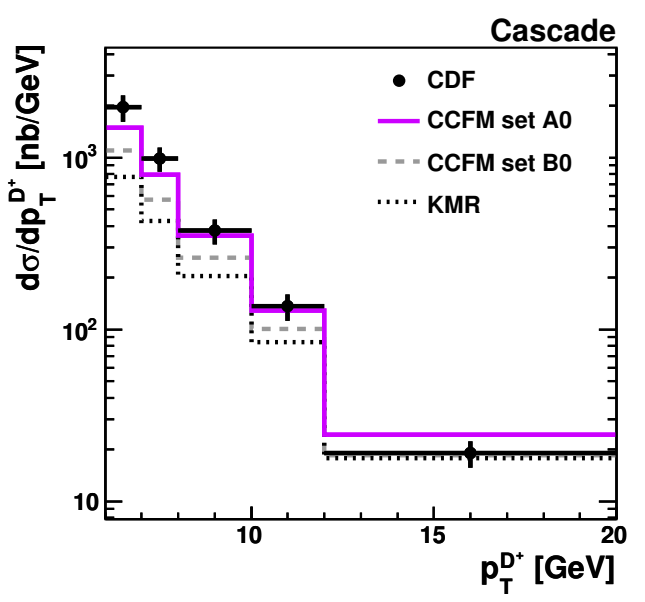

(d)

Figure 5. The cross section as a function of transverse momentum of $D^{0}[(\mathrm{a}),(\mathrm{b})]$ and $D^{+}[(\mathrm{c}),(\mathrm{d})]$ meson hadroproduction. The kinematical cuts applied are described in the text. The first column shows the LZ numerical results while the second one depicts the CASCADE predictions. Notation of all histograms is the same as in figure 3. The experimental data are from CDF [5].

$m_{c}=1.4 \mathrm{GeV}$ by $\pm 0.1 \mathrm{GeV}$. All heavy flavour cross sections are observed to be lower, if the masses are enlarged and vice versa.

We turn now to the investigation of the angular correlations between the produced particles. As it was mentioned above, such correlations have been not studied yet in the framework of GM-VFNS scheme (which has been applied for transverse momentum distributions of the charm and beauty mesons only [10, 11]). Experimental data on the azimuthal correlations in charm and beauty production come from both the CDF and D0 collaborations. In the case of $b$-quark production, CDF data [3] refer to the $B, \bar{B}$ azimuthal angle distribution measured at $|y|<1, p_{T}(B)>14 \mathrm{GeV}, p_{T}(\bar{B})>7.5 \mathrm{GeV}$ and $\sqrt{s}=1800 \mathrm{GeV}$. The D0 data [1] refer to the muon-muon correlation measured in the region of $4<p_{T}^{\mu}<25 \mathrm{GeV},\left|\eta^{\mu}\right|<0.8$ and $6<m^{\mu \mu}<35 \mathrm{GeV}$ at the same energy. In the 


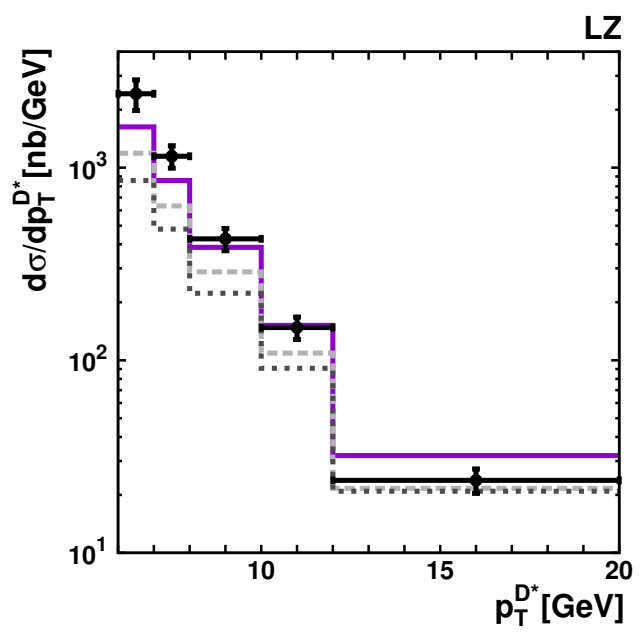

(a)

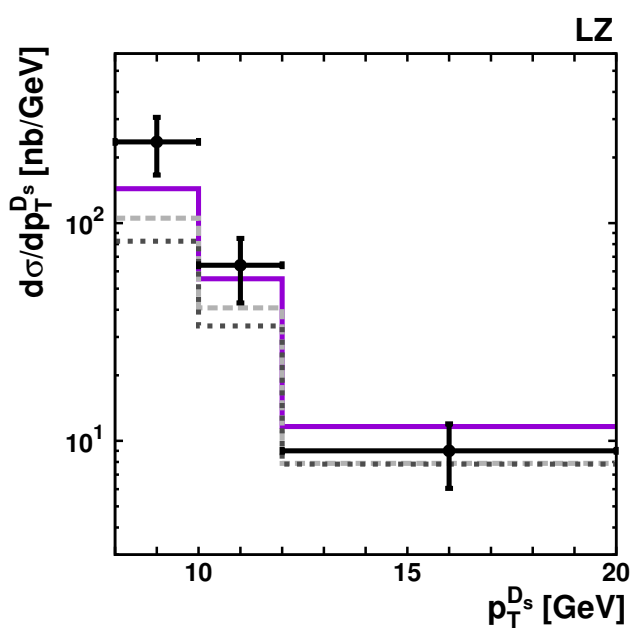

(c)

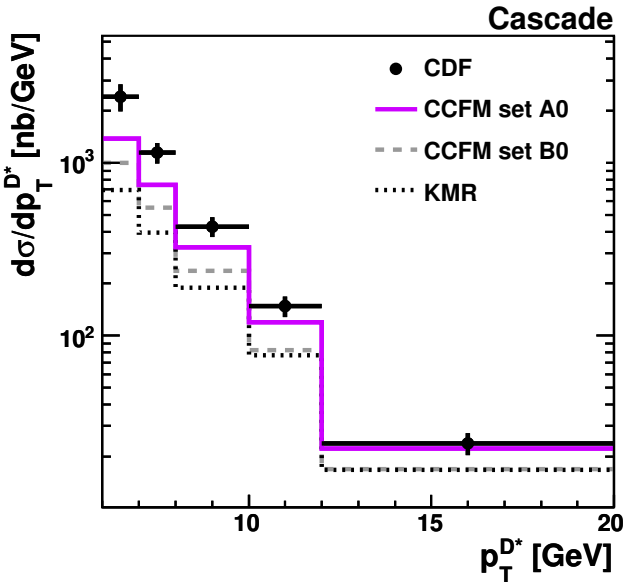

(b)

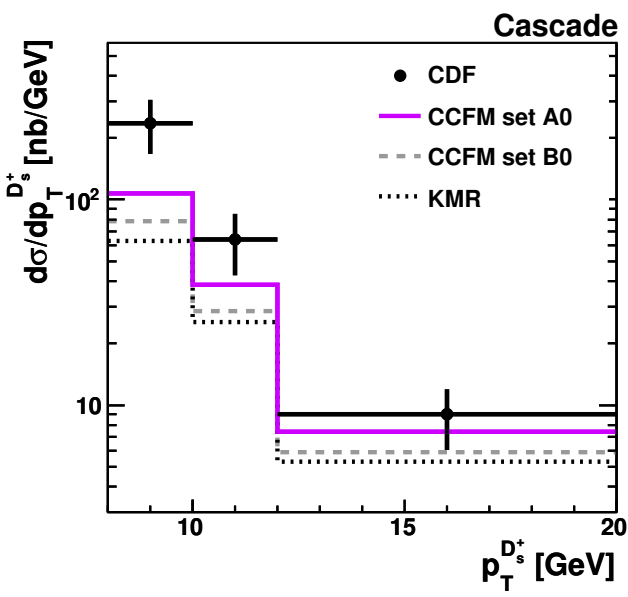

(d)

Figure 6. The cross section as a function of the transverse momentum of $D^{*}[(\mathrm{a}),(\mathrm{b})]$ and $D_{s}^{+}$ $[(\mathrm{c}),(\mathrm{d})]$ meson hadroproduction. The kinematical cuts applied are described in the text. The first column shows the LZ numerical results while the second one depicts the CASCADE predictions. Notation of all histograms is the same as in figure 3. The experimental data are from CDF [5].

case of charm production, the CDF collaboration has presented the measurement [6] of $D^{0}$, $D^{*-}$ and $D^{+}, D^{*-}$ pair cross section as a function of angle between the two charm mesons for the kinematic range $|y|<1,5.5<p_{T}\left(D^{0}\right)<20 \mathrm{GeV}, 5.5<p_{T}\left(D^{*-}\right)<20 \mathrm{GeV}$ and $7<p_{T}\left(D^{+}\right)<20 \mathrm{GeV}$. Our predictions are shown in figures 10 and 11 in comparison with the data $[1,3,6]$. We observe that the predicted shapes of azimuthal angle distributions are very different for different unintegrated gluon density functions. This is in a contrast to the cross sections as a function of transverse momenta or rapidities where all unintegrated gluon densities gave a similar behaviour. We can conclude that the cross section as a function of $\Delta \phi$ is very sensitive to the details of the non-collinear gluon evolution in a proton. As it was pointed out in $[12,17,18]$, such observables can serve as an important 


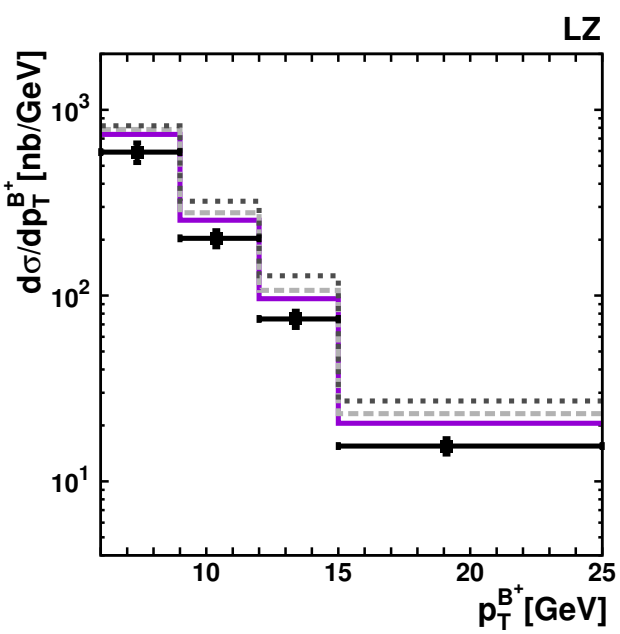

(a)

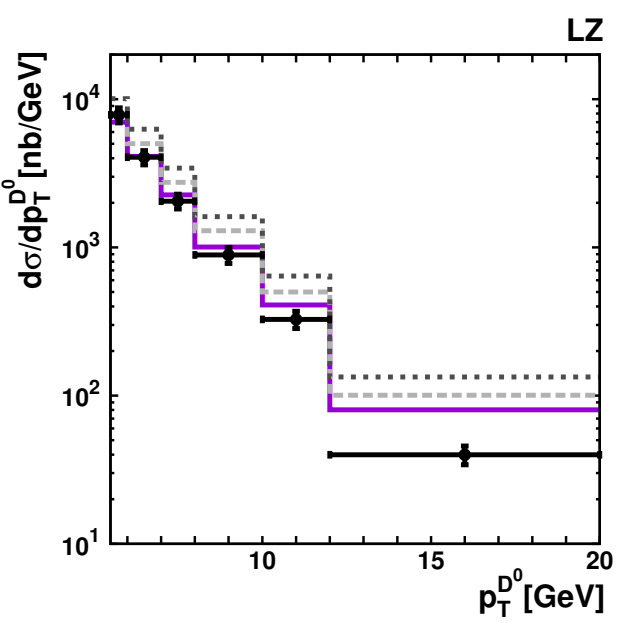

(c)

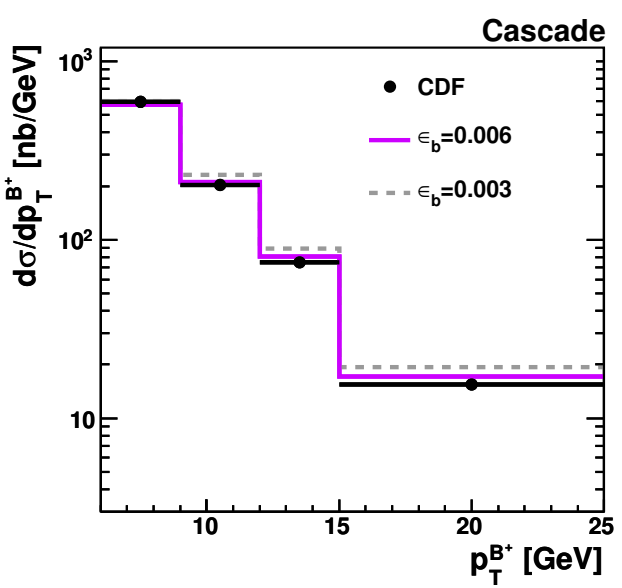

(b)

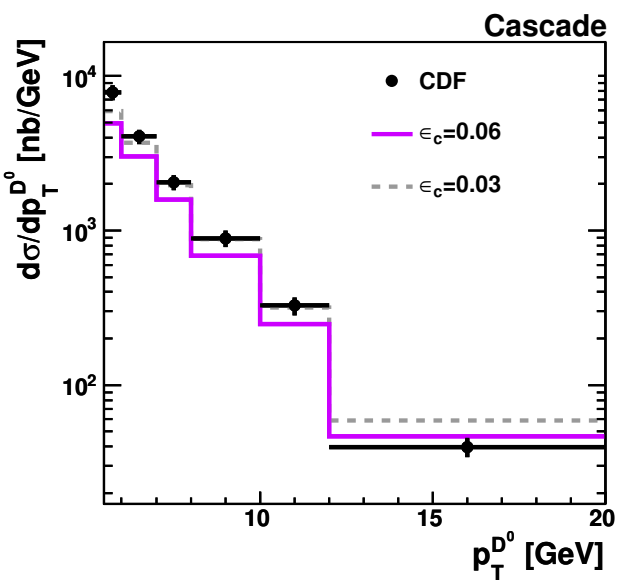

(d)

Figure 7. The dependence of our predictions on the fragmentation scheme applied. The soild, dashed and dotted histograms correspond to the results obtained using the Peterson fragmentation function with $\epsilon_{b}=0.006\left(\epsilon_{c}=0.06\right), \epsilon_{b}=0.003\left(\epsilon_{c}=0.03\right)$ and the non-perturbative fragmentation functions from $[7,9,50]$. The first column shows the LZ numerical results while the second one depicts the CASCADE predictions. Here we use CCFM set A0 gluon density for illustration. The experimental data are from $\mathrm{CDF}[4,5]$.

and crucial test discriminating the different approaches of the small- $x$ physics. The CCFMevolved gluon densities overshoot the data at $\Delta \phi \sim \pi$ and tends to underestimate them at $\Delta \phi \sim 0$. We observe that the peak at $\Delta \phi \rightarrow 0$ is not at all reproduced by the CCFM unintegrated gluon densities; we address this point in more detail in section 3.3 where we discuss the the process $g g^{*} \rightarrow g g$. However the KMR gluon density has obviously a very different $k_{T}$ distribution (see figure 1) and therefore provides a better description.

\section{$3.2 \quad b \bar{b}$ di-jet production}

Recently, the experimental study of the $b \bar{b}$ di-jet production in $p \bar{p}$ collisions at $\sqrt{s}=$ $1960 \mathrm{GeV}$ has been presented [33, 34]. The total cross section has been measured in the kinematical region defined by $\left|y_{1}\right|<1.2,\left|y_{2}\right|<1.2, E_{1 T}>35 \mathrm{GeV}$ and $E_{2 T}>32 \mathrm{GeV}$. The 


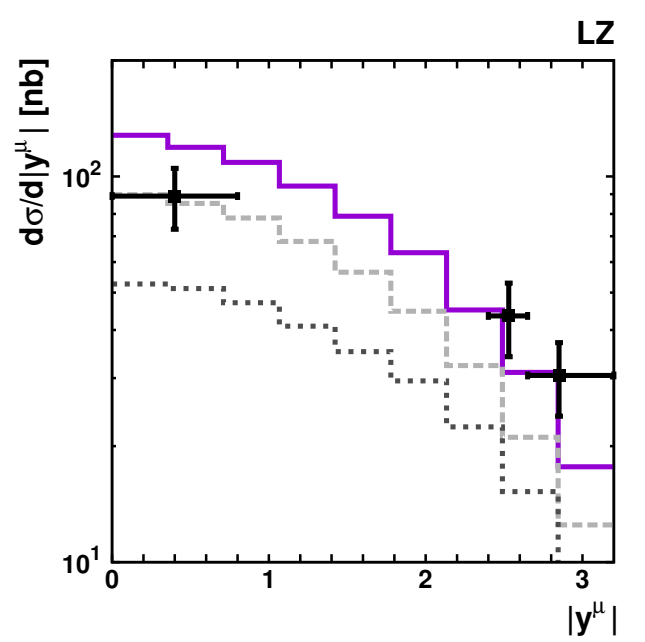

(a)

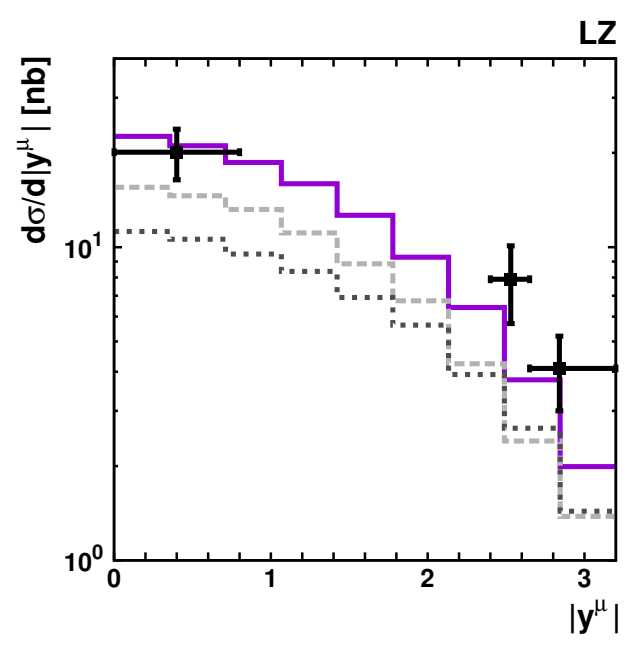

(c)

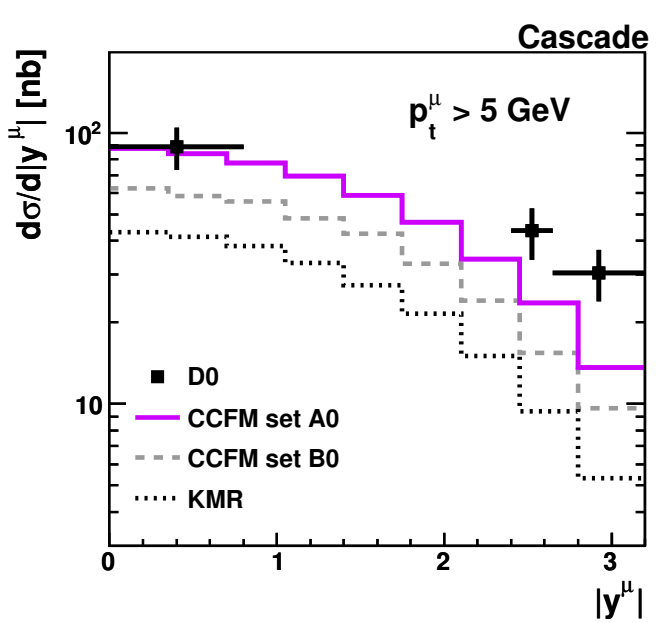

(b)

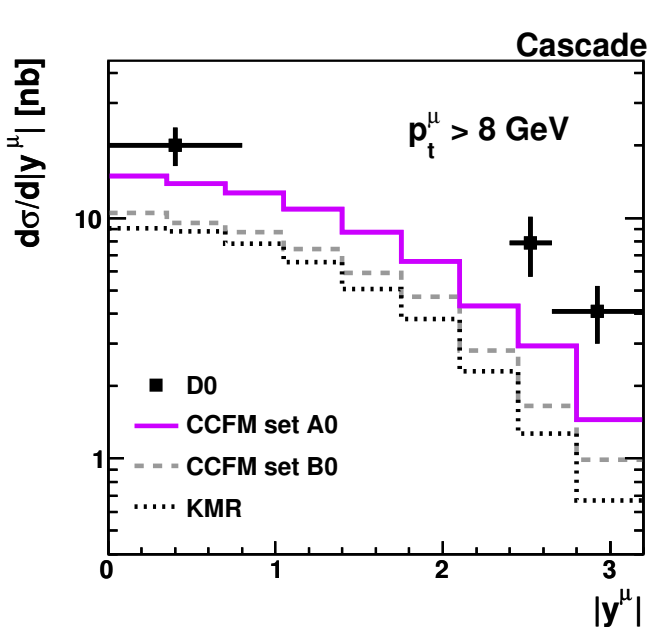

(d)

Figure 8. The rapidity distributions of muons arising from the semileptonic decays of $B$-mesons. The first column shows the LZ numerical results while the second one depicts the CASCADE predictions. The kinematical cuts applied are described in the text. Notation of all histograms is the same as in figure 3. The experimental data are from D0 [2].

differential cross sections as a functions of the leading jet transverse energy and of the di-jet invariant mass have been measured with the azimuthal angle correlation between the two jets. The $b$-jets are reconstructed with the JETCLU cone algorithm with radius $R>0.4$. Our predictions are shown in figures $12-14$. We observe that at large $E_{T}$ and at large dijet invariant masses $M$ the predictions fall below the measurement. Note, however, that in this kinematical region the quark induced subprocesses (such as $q \bar{q} \rightarrow b \bar{b}$ ) becomes important but are not taken into account here. At small and moderate $E_{T}$ and $M$, where gluon induced subprocesses play the leading role, the overall agreement of our predictions and the data is quite good. The measured $\Delta \phi$ distribution has a significantly different tail at small $\Delta \phi$ compared to the predictions; this will be addressed in more detail in the next section. 


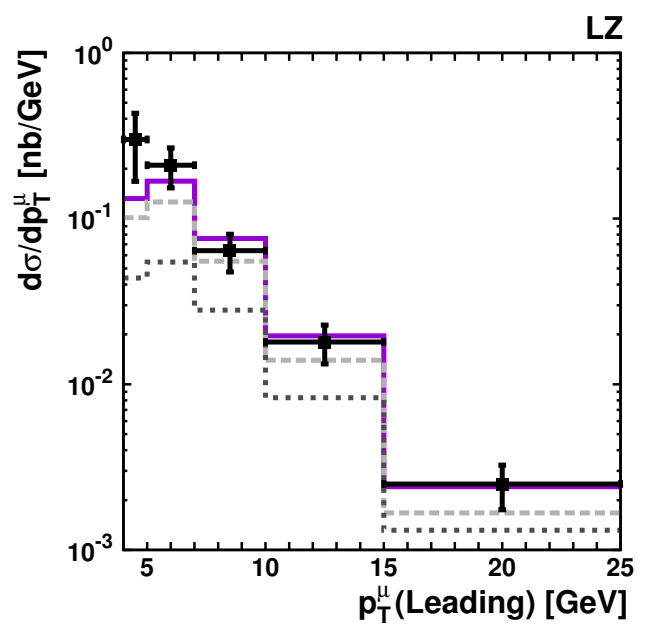

(a)

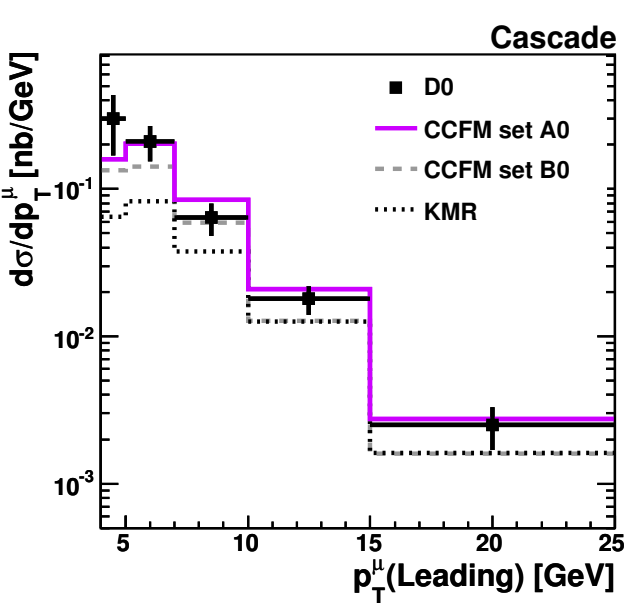

(b)

Figure 9. The transverse momentum distributions of muons arising from the semileptonic decays of $B$-mesons. The left histogram shows the LZ numerical results while the right plot depicts the CASCADE predictions. The kinematical cuts applied are described in the text. Notation of all histograms is the same as in figure 3. The experimental data are from D0 [1].

\begin{tabular}{|l|c|c|c|c|c|}
\hline Source & $\sigma\left(B^{+}\right)$ & $\sigma\left(D^{0}\right)$ & $\sigma\left(D^{+}\right)$ & $\sigma\left(D^{*+}\right)$ & $\sigma\left(D_{s}^{+}\right)$ \\
\hline CDF data $[\mu \mathrm{b}]$ & $2.78 \pm 0.24$ & $13.3 \pm 1.5$ & $4.3 \pm 0.7$ & $5.2 \pm 0.8$ & $0.75 \pm 0.23$ \\
\hline A0 (LZ/CASCADE) & $3.47 / 2.76$ & $13.34 / 9.31$ & $4.53 / 3.45$ & $3.81 / 3.19$ & $0.49 / 0.35$ \\
B0 (LZ/CASCADE) & $2.54 / 2.02$ & $9.55 / 6.78$ & $3.27 / 2.54$ & $2.78 / 2.32$ & $0.35 / 0.26$ \\
KMR (LZ/CASCADE) & $1.33 / 1.16$ & $6.92 / 5.06$ & $2.40 / 1.92$ & $2.13 / 1.75$ & $0.29 / 0.22$ \\
KMR (LZ, MSTW 2008) & 1.16 & 6.04 & 2.17 & 1.79 & 0.25 \\
\hline
\end{tabular}

Table 1. The charm and beauty total cross section in $p \bar{p}$ collisions at $\sqrt{s}=1960 \mathrm{GeV}$. The measurement $[4,5]$ was obtained in the central rapidity region $(|y|<1)$ at $6<p_{T}<25 \mathrm{GeV}$ and $5.5<p_{T}<20 \mathrm{GeV}$ in the case of $B$ and $D$ mesons, respectively.

\subsection{Parton shower effects and additional gluon processes}

As shown above, the full set of the data on the heavy flavour production is in general reasonably well described. However the measured cross sections as a function of $\Delta \phi$ show significant differences. These distributions are directly sensitive to additional parton ra- 


\begin{tabular}{|l|c|c|c|c|c|}
\hline Source & $\sigma\left(B^{+}\right)$ & $\sigma\left(D^{0}\right)$ & $\sigma\left(D^{+}\right)$ & $\sigma\left(D^{*+}\right)$ & $\sigma\left(D_{s}^{+}\right)$ \\
\hline CCFM set A0 & 2.76 & 9.31 & 3.45 & 3.19 & 0.35 \\
CCFM set A0+ & $+10 \%$ & $+6 \%$ & $+6 \%$ & $+5 \%$ & $+9 \%$ \\
CCFM set A0- & $+1 \%$ & $+4 \%$ & $+4 \%$ & $+3 \%$ & $+3 \%$ \\
$m_{b}=5.0 \mathrm{GeV}, m_{c}=1.5 \mathrm{GeV}$ & $-8 \%$ & $-4 \%$ & $-4 \%$ & $-4 \%$ & $-3 \%$ \\
$m_{b}=4.5 \mathrm{GeV}, m_{c}=1.3 \mathrm{GeV}$ & $+11 \%$ & $+4 \%$ & $+1 \%$ & $+4 \%$ & $+11 \%$ \\
$\epsilon_{b}=0.003, \epsilon_{c}=0.03$ & $+7 \%$ & $+21 \%$ & $+23 \%$ & $+21 \%$ & $+25 \%$ \\
\hline Total & $\pm_{8 \%}^{16 \%}$ & $\pm_{4 \%}^{23 \%}$ & $\pm_{4 \%}^{24 \%}$ & $\pm_{4 \%}^{22 \%}$ & $\pm_{3 \%}^{29 \%}$ \\
\hline
\end{tabular}

Table 2. Systematic uncertainties for charm and beauty total cross sections in $p \bar{p}$ collisions at $\sqrt{s}=1960 \mathrm{GeV}$ obtained with CASCADE. The measurement $[4,5]$ was obtained in the central rapidity region $(|y|<1)$ at $6<p_{T}<25 \mathrm{GeV}$ and $5.5<p_{T}<20 \mathrm{GeV}$ in the case of $B$ and $D$ mesons, respectively.

diation treated by parton showers or by other additional processes. In the following we investigate the influence of the details of the parton shower and the process $g g^{*} \rightarrow g g$ with subsequent branching of one of the outgoing (off-shell) $g \rightarrow b \bar{b}$ on the $\Delta \phi$ distribution. For these studies we use the CCFM set A0.

In CASCADE, the initial state parton shower is angular ordered and based on the CCFM evolution equations. The final state parton shower is also angular ordered, but based on the DGLAP evolution equations. To investigate the dependence on the parton shower, we calculated the cross sections without parton shower, only initial state, only final state and both initial and final state parton shower, as shown in figure 15. Here, $b$ dijet production is selected as an example. We observe a very small contribution of the initial state parton shower, since in $k_{T}$-factorisation the initial state parton shower does not influence the $k_{T}$ of the gluons (since it is determined from the unintegrated gluon density). However, a significant contribution comes from the final state parton shower. The prediction with full parton shower underestimates the back-to-back region of the azimuthal separation of the two $b$ jets. This suggests that the final state parton showering generates too much gluon radiation, which causes less correlated jets as shown in figure 15 (a). To investigate this further we changed the final state parton shower scale $Q_{\max }^{2}$ from $Q_{\max }^{2}=4 m_{T}^{2}$ to $Q_{\max }^{2}=m^{2}$. As shown in figure $15(\mathrm{~b})$, this leads to a higher correlation of the $b$ jets and a very good description of the back-to-back region of the jets is achieved. 


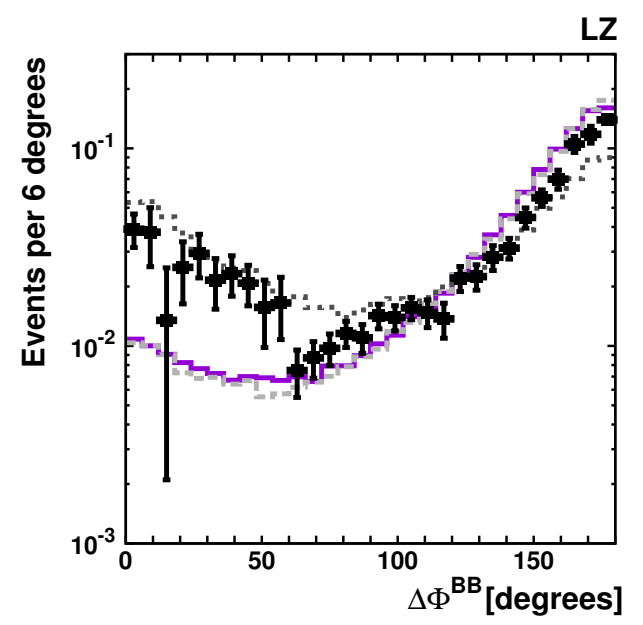

(a)

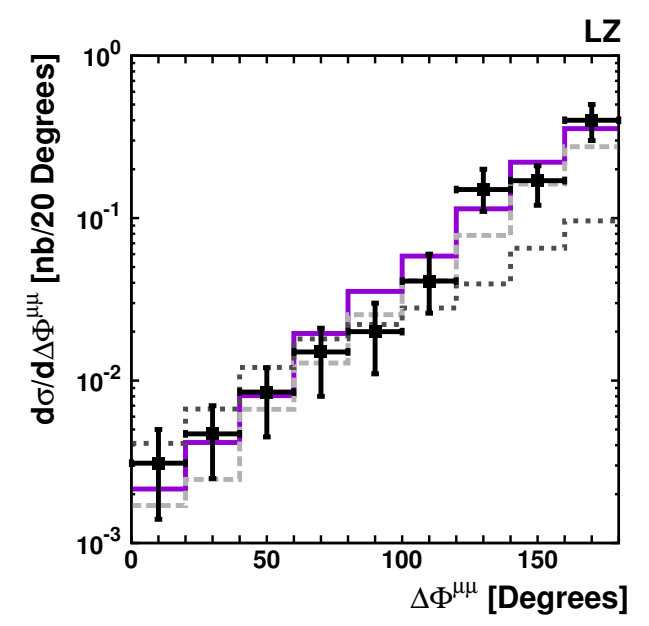

(c)

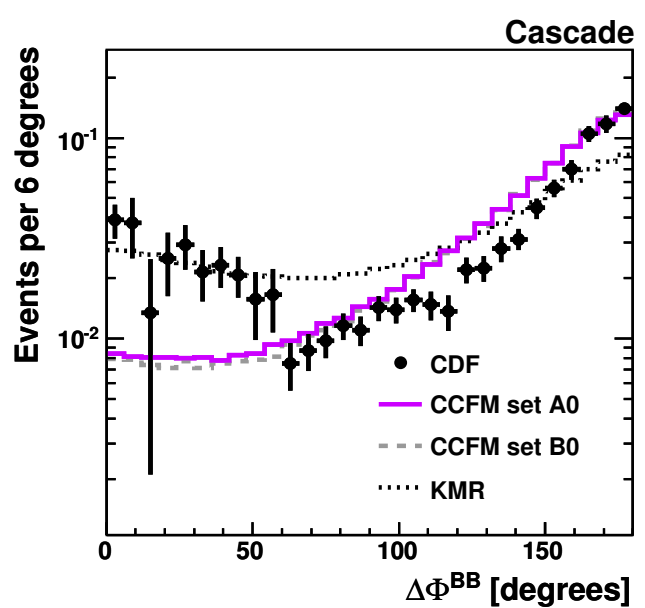

(b)

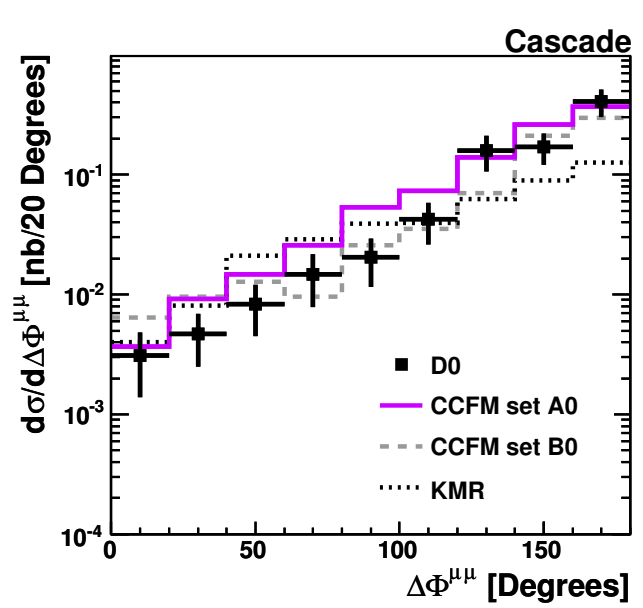

(d)

Figure 10. The distributions in azimuthal angle difference between the produced $B$-mesons [(a), (b)] and muons [(c), (d)], originating from their semileptonic decays. The first column shows the LZ numerical results while the second one depicts the CASCADE predictions. The kinematical cuts applied are described in the text. Notation of all histograms is the same as in figure 3 . The experimental data are from CDF [3] and D0 [1].

At lower values of the azimuthal separation of the dijet system, higher order processes are expected to contribute significantly. Therefore, we repeated the $b$ dijet analysis for the process $g g^{*} \rightarrow g g$. In the matrix element calculation, which was performed in [51], one gluon in the initial state is on-shell and the other one is off-shell, as shown in figure 16. The heavy quark pair is then produced via parton showers in the final state as $g \rightarrow b \bar{b}$. As shown in figure 17 (a), this process contributes significantly to the tail of the $\Delta \Phi_{j j}$ distribution very well, without any additional adjustment of parameters.

In conclusion, the azimuthal cross section measurements can be very well described by adjusting the scale for the final state parton shower to $Q_{\max }^{2}=m^{2}$ and by including the process $g g^{*} \rightarrow g g$ with subsequent branching $g \rightarrow b \bar{b}$, which in a collinear calculation would contribute only at NNLO. 


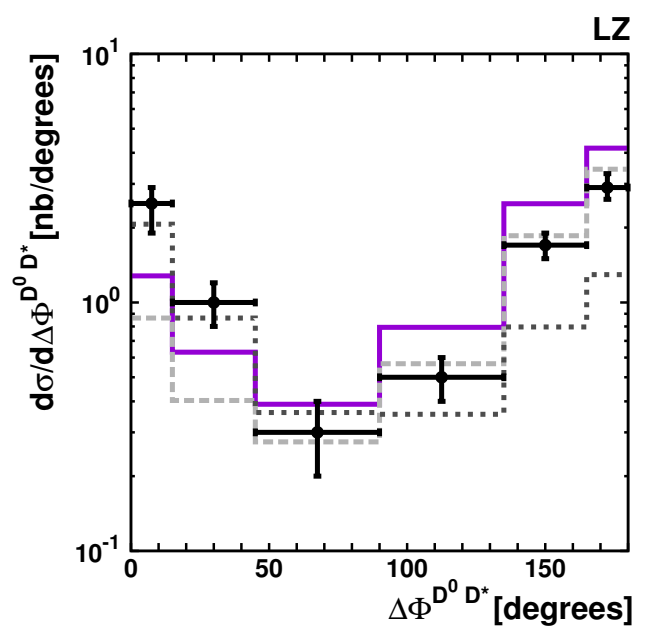

(a)

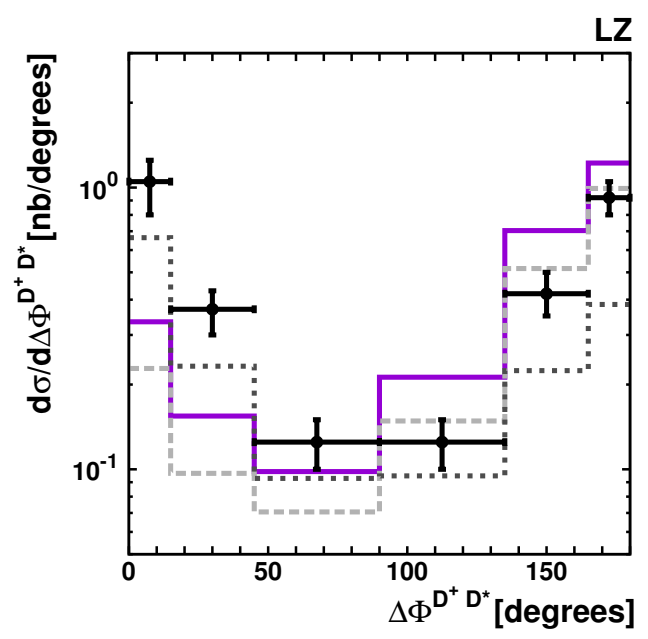

(c)

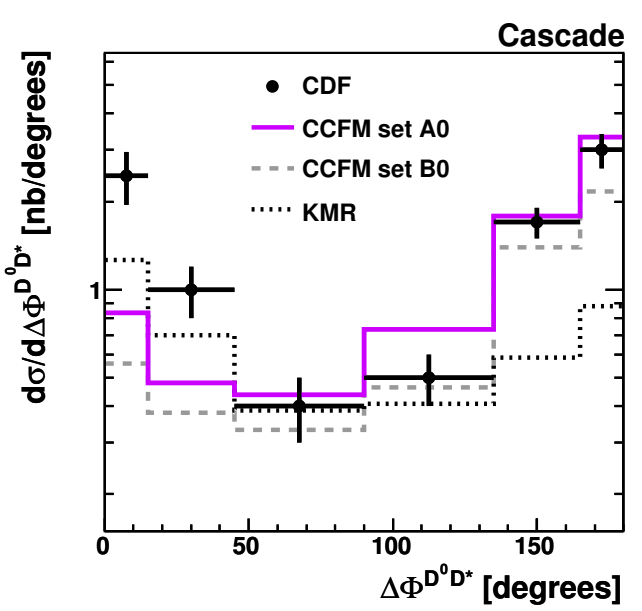

(b)

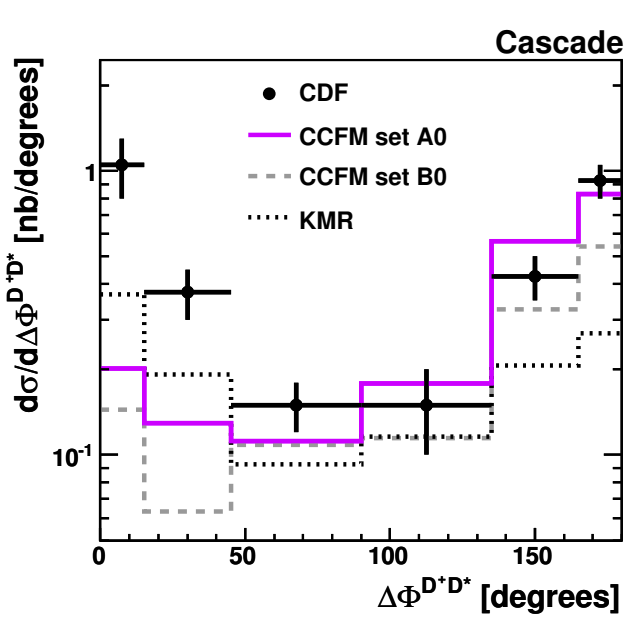

(d)

Figure 11. The distributions in azimuthal angle difference between the produced $D^{0}, D^{*-}$ and $D^{+}, D^{*-}$ mesons. The first column shows the LZ numerical results while the second one depicts the CASCADE predictions. The cuts applied are described in the text. Notation of all histograms is the same as in figure 3. The experimental data are from CDF [6].

\section{Conclusions}

We have studied charm and beauty production at Tevatron energies within the framework of the $k_{T}$-factorization. Our calculations are based on the CCFM-evolved unintegrated gluon densities in a proton. The analysis covers the total and differential cross sections of open charm and beauty quarks, $B$ and $D$ mesons (or rather muons from their semileptonic decays). The cross sections of $b \bar{b}$ di-jet production have been studied also. Special attention was put on the specific angular correlations between the final-state particles. Using full hadron-level Monte Carlo generator CASCADE, we investigated the effects coming from the parton showers in initial and final states. Different sources of theoretical uncertainties have been specially studied. 


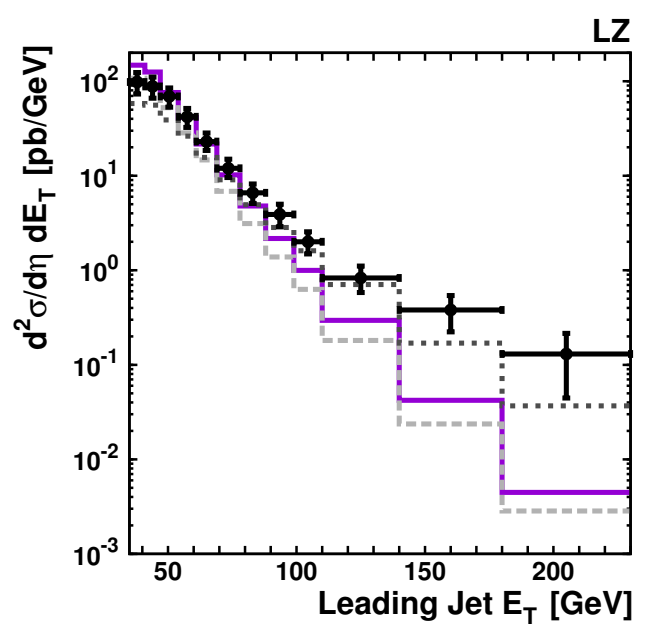

(a)

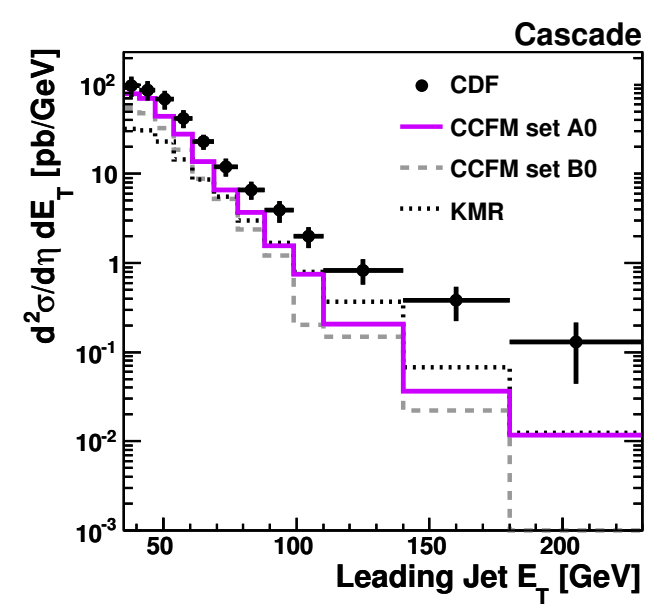

(b)

Figure 12. The leading jet transverse energy distributions of $b \bar{b}$ di-jet production. The left histogram shows the LZ numerical results while the right plot depicts the CASCADE predictions. The kinematical cuts applied are described in the text. Notation of all histograms is the same as in figure 3. The experimental data are from $\operatorname{CDF}[33,34]$.

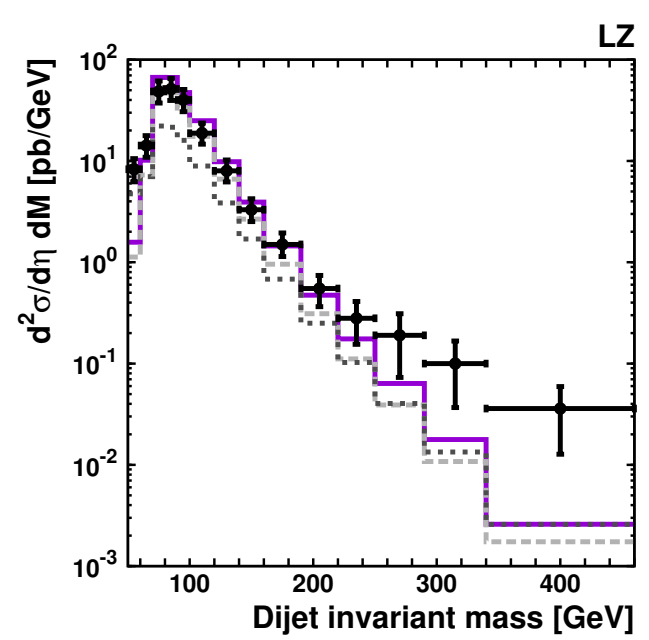

(a)

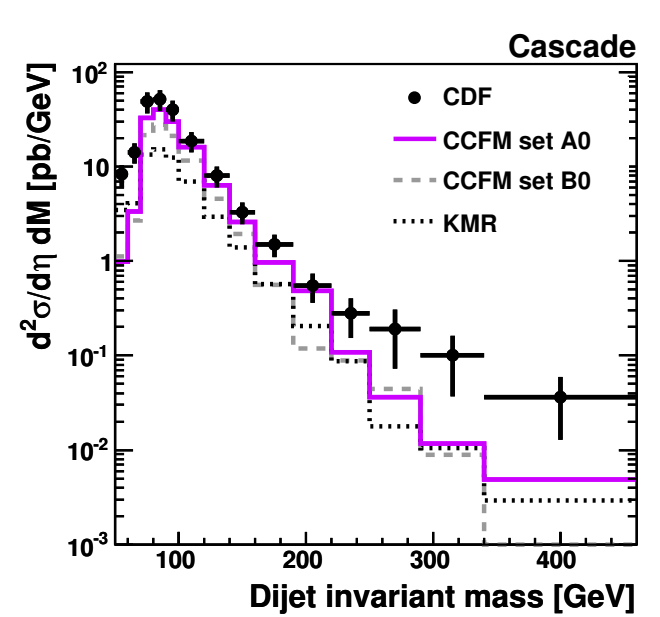

(b)

Figure 13. The invariant mass distributions of $b \bar{b}$ di-jet production. The left histogram shows the LZ numerical results while the right plot depicts the CASCADE predictions. The kinematical cuts applied are described in the text. Notation of all histograms is the same as in figure 3. The experimental data are from $\mathrm{CDF}[33,34]$.

We obtain good agreement of our calculations for all observables and the recent experimental data taken by the D0 and CDF collaborations. We have demonstrated, that the parton shower plays a significant role in the description of the cross section. We obtain a good description of the correlations once the higher order process $g g^{*} \rightarrow g g$ with subsequent $g \rightarrow b \bar{b}$ splitting is included. 


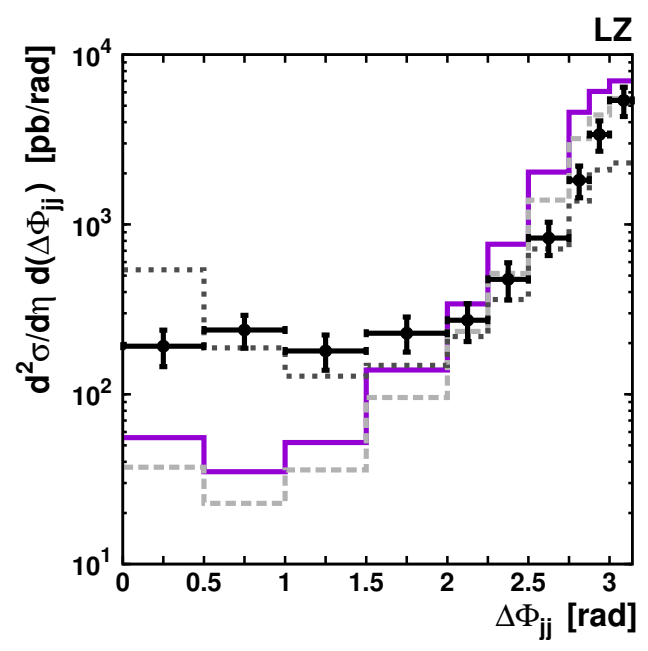

(a)

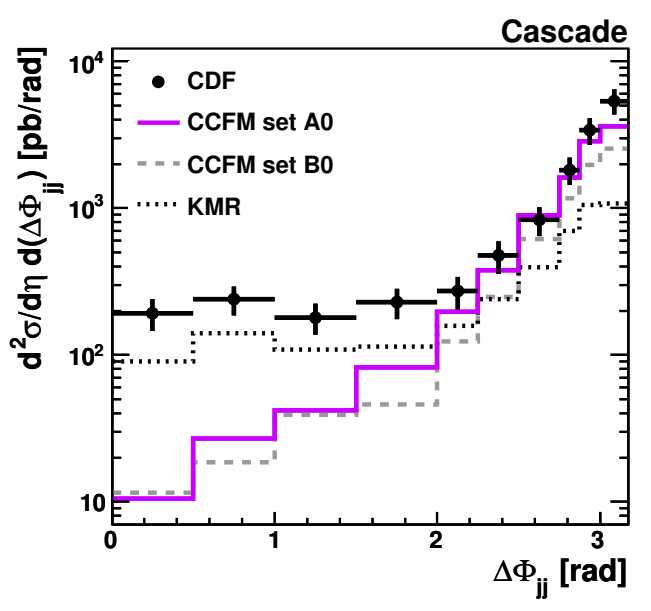

(b)

Figure 14. The distributions in azimuthal angle difference between the produced $b$-jets. The left histogram shows the LZ numerical results while the right plot depicts the CASCADE predictions. The kinematical cuts applied are described in the text. Notation of all histograms is the same as in figure 3. The experimental data are from $\mathrm{CDF}[33,34]$.

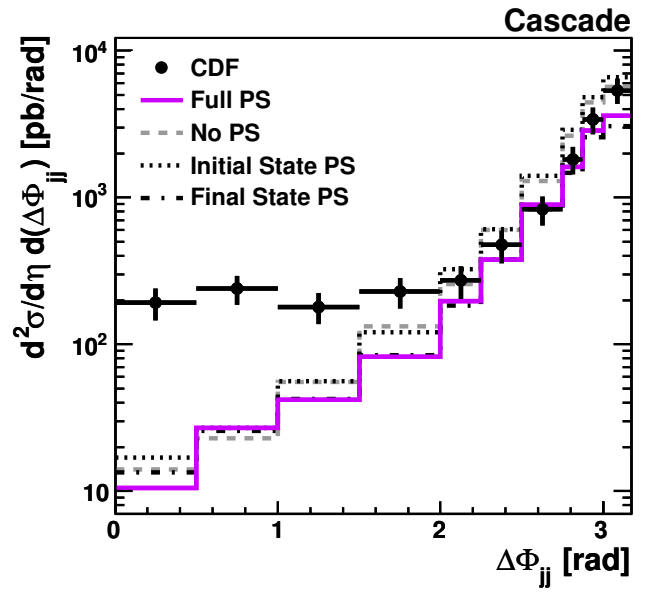

(a)

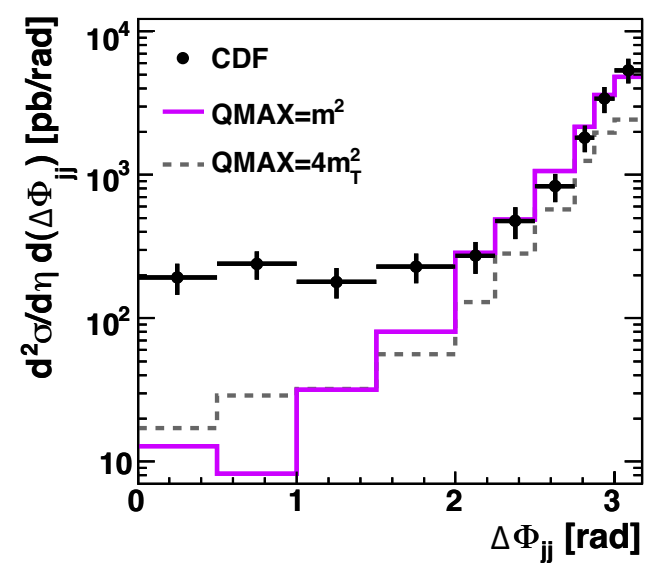

(b)

Figure 15. Parton shower effects for $b$ dijet production in (a). The four lines represent full parton shower (solid line), no parton shower (dashed line), initial state parton shower (dashed dotted line) and final state parton shower (dotted line). The change of the parton shower scale from $Q_{\max }=4 m_{T}^{2}$ to $Q_{\max }=m^{2}$ for $b$ dijet production is depicted in (b). In all cases the CCFM set A0 is used and the data are taken from $\mathrm{CDF}[33,34]$ and $\mathrm{CDF}$ [6].

\section{Acknowledgments}

We thank S.P. Baranov and A. Geiser for his encouraging interest and useful discussions. We are grateful to S. Vallecorsa for many explanations on the B-jet analysis of CDF. The authors are very grateful to DESY Directorate for the support in the framework of Moscow - DESY project on Monte-Carlo implementation for HERA - LHC. A.V.L. was 


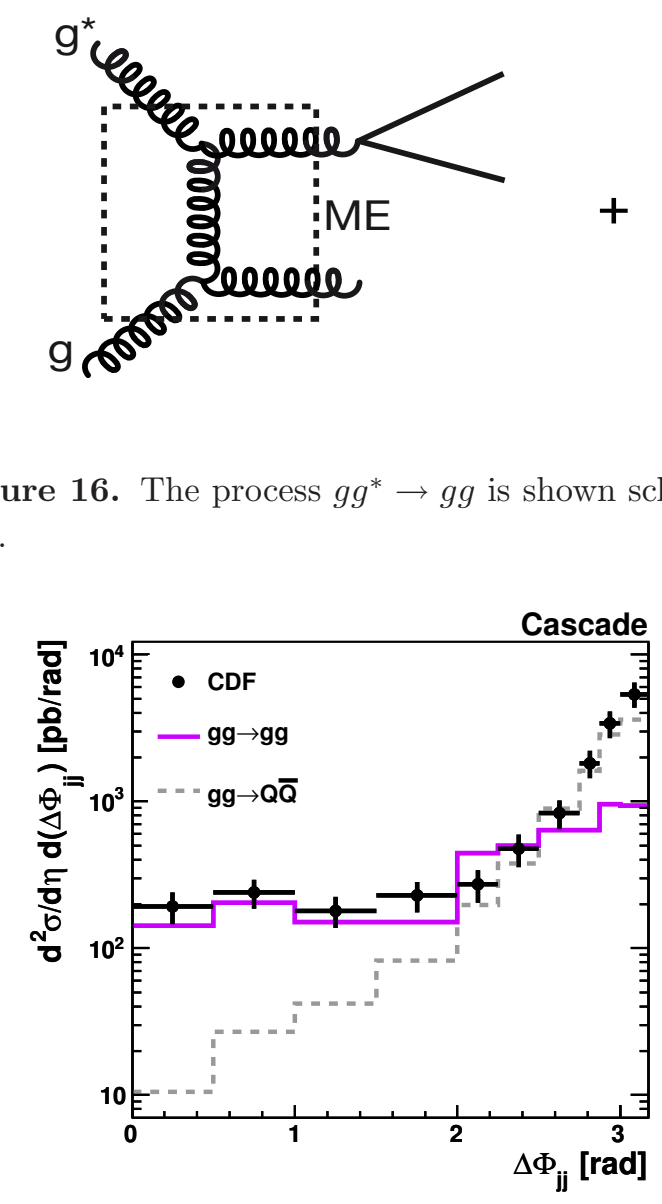

(a)

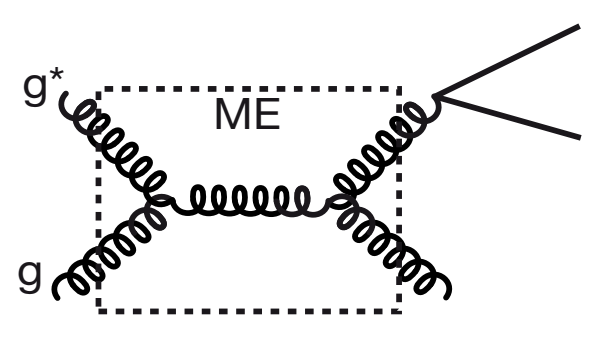

(a)

Figure 16. The process $g g^{*} \rightarrow g g$ is shown schematically. The matrix elements are calculated in [51].

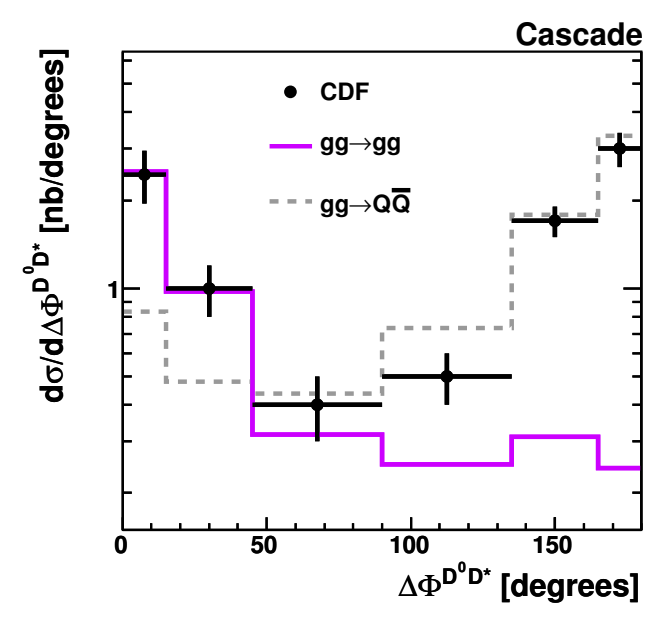

(b)

Figure 17. The processes $g^{*} g^{*} \rightarrow Q \bar{Q}$ (dashed line) and $g g^{*} \rightarrow g g$ for $b$ dijet production in (a) and $D^{0}, D^{*-}$ meson production in (b). In all cases the CCFM set A0 is used and the data are taken from $\mathrm{CDF}[33,34]$ and $\mathrm{CDF}[6]$.

supported in part by the Helmholtz - Russia Joint Research Group. Also this research was supported by the FASI of Russian Federation (grant NS-4142.2010.2) and FASI state contract 02.740.11.0244.

Open Access. This article is distributed under the terms of the Creative Commons Attribution Noncommercial License which permits any noncommercial use, distribution, and reproduction in any medium, provided the original author(s) and source are credited.

\section{References}

[1] D0 collaboration, B. Abbott et al., The b $\bar{b}$ production cross section and angular correlations

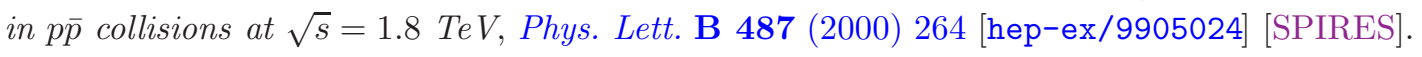

[2] D0 collaboration, B. Abbott et al., Small angle muon and bottom quark production in $p \bar{p}$ collisions at $\sqrt{s}=1.8 \mathrm{TeV}$, Phys. Rev. Lett. 84 (2000) 5478 [hep-ex/9907029] [SPIRES]. 
[3] CDF collaboration, D.E. Acosta et al., Measurements of b $\bar{b}$ azimuthal production correlations in py collisions at $\sqrt{s}=1.8$ TeV, Phys. Rev. D 71 (2005) 092001 [hep-ex/0412006] [SPIRES].

[4] CDF collaboration, A. Abulencia et al., Measurement of the B+ production cross section in $p \bar{p}$ collisions at $\sqrt{s}=1960$ GeV, Phys. Rev. D 75 (2007) 012010 [hep-ex/0612015] [SPIRES].

[5] CDF collaboration, D.E. Acosta et al., Measurement of prompt charm meson production cross sections in p $\bar{p}$ collisions at $\sqrt{s}=1.96$ TeV, Phys. Rev. Lett. 91 (2003) 241804 [hep-ex/0307080] [SPIRES].

[6] J. Rademacker, Proceedings of Charm '07, Ithaca, New York, U.S.A. (2007).

[7] M. Cacciari and P. Nason, Is there a significant excess in bottom hadroproduction at the Tevatron?, Phys. Rev. Lett. 89 (2002) 122003 [hep-ph/0204025] [SPIRES].

[8] M. Cacciari, S. Frixione, M.L. Mangano, P. Nason and G. Ridolfi, QCD analysis of first b cross-section data at $1.96 \mathrm{TeV}$, JHEP 07 (2004) 033 [hep-ph/0312132] [SPIRES].

[9] M. Cacciari and P. Nason, Charm cross-sections for the Tevatron Run II, JHEP 09 (2003) 006 [hep-ph/0306212] [SPIRES].

[10] B.A. Kniehl, G. Kramer, I. Schienbein and H. Spiesberger, Finite-mass effects on inclusive B meson hadroproduction, Phys. Rev. D 77 (2008) 014011 [arXiv: 0705.4392] [SPIRES].

[11] B.A. Kniehl, G. Kramer, I. Schienbein and H. Spiesberger, Open charm hadroproduction and the charm content of the proton, Phys. Rev. D 79 (2009) 094009 [DESY-09-008] [arXiv:0901.4130] [SPIRES].

[12] S.P. Baranov and M. Smizanska, Semihard b quark production at high-energies versus data and other approaches, Phys. Rev. D 62 (2000) 014012 [SPIRES].

[13] P. Hagler, R. Kirschner, A. Schafer, L. Szymanowski and O. Teryaev, Heavy quark production as sensitive test for an improved description of high-energy hadron collisions, Phys. Rev. D 62 (2000) 071502 [hep-ph/0002077] [SPIRES].

[14] M.G. Ryskin, A.G. Shuvaev and Y.M. Shabelski, Comparison of $k(T)$ factorization approach and QCD parton model for charm and beauty hadroproduction, Phys. Atom. Nucl. 64 (2001) 1995 [hep-ph/0007238] [SPIRES].

[15] Y.M. Shabelski and A.G. Shuvaev, Heavy quark hadroproduction in $k_{T}$-factorization approach with unintegrated gluon distributions, Phys. Atom. Nucl. 69 (2006) 314 [hep-ph/0406157] [SPIRES].

[16] N.P. Zotov, A.V. Lipatov and V.A. Saleev, Heavy-quark production in p p $\bar{p}$ collisions and unintegrated gluon distributions, Phys. Atom. Nucl. 66 (2003) 755 [SPIRES].

[17] S.P. Baranov, N.P. Zotov and A.V. Lipatov, Production of heavy quarks on protons within the semihard QCD approach, Phys. Atom. Nucl. 67 (2004) 837 [SPIRES].

[18] A.V. Lipatov, L. Lönnblad and N.P. Zotov, Study of the linked dipole chain model in heavy quark production at the Tevatron, JHEP 01 (2004) 010 [hep-ph/0309207] [SPIRES].

[19] L.V. Gribov, E.M. Levin and M.G. Ryskin, Semihard Processes in $Q C D$, Phys. Rept. 100 (1983) 1 [SPIRES].

[20] E.M. Levin, M.G. Ryskin, Y.M. Shabelski and A.G. Shuvaev, Heavy quark production in semihard nucleon interactions, Sov. J. Nucl. Phys. 53 (1991) 657 [SPIRES]/ 
[21] S. Catani, M. Ciafaloni and F. Hautmann, High-energy factorization and small $x$ heavy flavor production, Nucl. Phys. B 366 (1991) 135 [SPIRES].

[22] J.C. Collins and R.K. Ellis, Heavy quark production in very high-energy hadron collisions, Nucl. Phys. B 360 (1991) 3 [SPIRES].

[23] E.A. Kuraev, L.N. Lipatov and V.S. Fadin, Multi-Reggeon Processes in the Yang-Mills Theory, Sov. Phys. JETP 44 (1976) 443 [SPIRES]/

[24] E.A. Kuraev, L.N. Lipatov and V.S. Fadin, The Pomeranchuk Singularity in Nonabelian Gauge Theories, Sov. Phys. JETP 45 (1977) 199 [SPIRES].

[25] I.I. Balitsky and L.N. Lipatov, The Pomeranchuk Singularity in Quantum Chromodynamics, Sov. J. Nucl. Phys. 28 (1978) 822 [SPIRES].

[26] M. Ciafaloni, Coherence Effects in Initial Jets at Small Q ${ }^{2} / s$, Nucl. Phys. B 296 (1988) 49 [SPIRES].

[27] S. Catani, F. Fiorani and G. Marchesini, QCD Coherence in Initial State Radiation, Phys. Lett. B 234 (1990) 339 [SPIRES].

[28] S. Catani, F. Fiorani and G. Marchesini, Small x Behavior of Initial State Radiation in Perturbative QCD, Nucl. Phys. B 336 (1990) 18 [SPIRES].

[29] G. Marchesini, QCD coherence in the structure function and associated distributions at small x, Nucl. Phys. B 445 (1995) 49 [hep-ph/9412327] [SPIRES].

[30] Small x collaboration, B. Andersson et al., Small x phenomenology: Summary and status, Eur. Phys. J. C 25 (2002) 77 [hep-ph/0204115] [SPIRES].

[31] Small x collaboration, J.R. Andersen et al., Small x phenomenology: Summary and status 2002, Eur. Phys. J. C 35 (2004) 67 [hep-ph/0312333] [SPIRES].

[32] Small x collaboration, J.R. Andersen et al., Small x Phenomenology: Summary of the 3rd Lund Small $x$ Workshop in 2004, Eur. Phys. J. C 48 (2006) 53 [hep-ph/0604189] [SPIRES].

[33] CDF collaboration, T. Aaltonen et al., $b-\bar{b}$ dijet production using SVT, CDF note 8939 [http://www-cdf.fnal.gov/physics/new/qcd/abstracts/bb_SVT_07.html].

[34] S. Vallecorsa, Measurement of the b-barb di-jet cross section at CDF, Ph.D. thesis no. 3916, Università di Genova, Genova, Italy (2007).

[35] H. Jung, Un-integrated uPDFs in CCFM, hep-ph/0411287 [SPIRES].

[36] H. Jung, The CCFM Monte Carlo generator CASCADE, Comput. Phys. Commun. 143 (2002) 100 [hep-ph/0109102] [SPIRES].

[37] H. Jung et al., The CCFM Monte Carlo generator CASCADE 2.2.0, Eur. Phys. J. C 70 (2010) 1237 [DESY-10-107] [arXiv:1008.0152] [SPIRES].

[38] F. Hautmann and H. Jung, Angular correlations in multi-jet final states from kt-dependent parton showers, JHEP 10 (2008) 113 [arXiv:0805.1049] [SPIRES].

[39] M.A. Kimber, A.D. Martin and M.G. Ryskin, Unintegrated parton distributions, Phys. Rev. D 63 (2001) 114027 [hep-ph/0101348] [SPIRES].

[40] G. Watt, A.D. Martin and M.G. Ryskin, Unintegrated parton distributions and inclusive jet production at HERA, Eur. Phys. J. C 31 (2003) 73 [hep-ph/0306169] [SPIRES].

[41] M. Gluck, E. Reya and A. Vogt, Photonic parton distributions, Phys. Rev. D 46 (1992) 1973 [SPIRES]. 
[42] M. Gluck, E. Reya and A. Vogt, Dynamical parton distributions of the proton and small $x$ physics, Z. Phys. C 67 (1995) 433 [SPIRES].

[43] A.D. Martin, W.J. Stirling, R.S. Thorne and G. Watt, Parton distributions for the LHC, Eur. Phys. J. C 63 (2009) 189 [arXiv:0901.0002] [SPIRES].

[44] A.D. Martin, R.G. Roberts, W.J. Stirling and R.S. Thorne, Parton distributions and the LHC: $W$ and $Z$ production, Eur. Phys. J. C 14 (2000) 133 [hep-ph/9907231] [SPIRES].

[45] G.P. Lepage, A New Algorithm for Adaptive Multidimensional Integration, J. Comput. Phys. 27 (1978) 192 [SPIRES].

[46] M.L. Mangano, P. Nason and G. Ridolfi, Heavy quark correlations in hadron collisions at next-to-leading order, Nucl. Phys. B 373 (1992) 295 [SPIRES].

[47] C. Peterson, D. Schlatter, I. Schmitt and P.M. Zerwas, Scaling Violations in Inclusive $e^{+} e^{-}$ Annihilation Spectra, Phys. Rev. D 27 (1983) 105 [SPIRES].

[48] ALEPH collaboration, R. Barate et al., Study of charm production in $Z$ decays, Eur. Phys. J. C 16 (2000) 597 [hep-ex/9909032] [SPIRES].

[49] Particle Data Group collaboration, K. Hagiwara et al., Review of particle physics, Phys. Rev. D 66 (2002) 010001 [SPIRES].

[50] E. Braaten, K.-m. Cheung, S. Fleming and T.C. Yuan, Perturbative QCD fragmentation functions as a model for heavy quark fragmentation, Phys. Rev. D 51 (1995) 4819 [hep-ph/9409316] [SPIRES].

[51] M. Deak, F. Hautmann, H. Jung and K. Kutak, Forward Jet Production at the Large Hadron Collider, JHEP 09 (2009) 121 [arXiv:0908.0538] [SPIRES]. 